Portland State University

PDXScholar

6-6-2021

\title{
Seabed Observations at the New England Mud Patch: Reflection and Scattering Measurements and Direct Geoacoustic Information
}

\author{
Charles W. Holland \\ Portland State University, hollan7@pdx.edu \\ Chad M. Smith \\ Penn State University \\ Zackary Lowe \\ Penn State University \\ Jim Dorminy \\ Penn State University
}

Follow this and additional works at: https://pdxscholar.library.pdx.edu/ece_fac

Part of the Electrical and Computer Engineering Commons

Let us know how access to this document benefits you.

\section{Citation Details}

Holland, C. W., Smith, C. M., Lowe, Z., \& Dorminy, J. (2021). Seabed Observations at the New England Mud Patch: Reflection and Scattering Measurements and Direct Geoacoustic Information. IEEE Journal of Oceanic Engineering, 1-16. https://doi.org/10.1109/joe.2021.3070028

This Post-Print is brought to you for free and open access. It has been accepted for inclusion in Electrical and Computer Engineering Faculty Publications and Presentations by an authorized administrator of PDXScholar. Please contact us if we can make this document more accessible: pdxscholar@pdx.edu. 


\author{
Seabed observations at the New England Mud Patch: reflection and \\ scattering measurements and direct geoacoustic information \\ Charles W. Holland ${ }^{1}$, Chad M. Smith ${ }^{2}$, Zackary Lowe ${ }^{2}$, Jim Dorminy ${ }^{2}$ \\ 1. Portland State University, Portland, OR \\ 2. The Pennsylvania State University, Applied Research Lab, State College, PA
}

\begin{abstract}
Seabed reflection and scattering measurements were conducted at the New England Mud Patch to better understand the acoustic properties of fine-grained (muddy) sediments. The measurement philosophy and the measurements themselves are summarized. In addition, geoacoustic information accessed directly from the data in the time and frequency domains are presented. The main result is the existence of an angle of intromission. This observation proves that the mud sound speed is less than that of the water and yields a sediment sound speed ratio 0.9865 with outer bounds $\{0.9850 .989\}$. Another result is the observation of strong scattered arrivals from within the mud volume at/near normal incidence but not at low grazing angles. These are likely due to anisotropic sediment heterogeneities with a large horizontal to vertical scale. Evidence is also presented for a highly heterogeneous mud-sand horizon with lateral variability down to scales of order meters. Finally, the reflection measurements successfully capture Bragg interference patterns. Their importance is their substantial geoacoustic information content, which can be accessed by several inversion methods.
\end{abstract}

\title{
I. Introduction
}

Marine and terrestrial life exploit sound to communicate and sense the ocean environment. Often, the seabed properties have a profound influence on sound propagation. The broad motivation for the multi-national Seabed Characterization Experiment, SBCEX17, was to better understand acoustic propagation through fine-grained, muddy sediments. Compared to granular (sandy) sediments, much less is known about the acoustics of fine-grained sediments. As one evidence of this, until very recently [1] there was no first principles model for sound propagation through fine-grained sediments, whereas for granular sediments, a model [2] has been in existence for six decades with a large number of variants and extensions. In accord with the paucity of models, there has been significantly fewer sediment acoustics experiments conducted in muddy than sandy sediments.

The primary long-term science objective of the research described here is to measure the intrinsic frequency-dependence of the sound speed and attenuation in muddy sediments from a few hundred hertz to a few kilohertz. Some discussion is warranted concerning experimentally inferred frequency dependence compared to that predicted by theoretical sediment acoustic models. Theoretical models of the frequency-dependence of sound speed and attenuation treat a homogeneous sample - at scales much larger than the individual grains. However, in an at-sea measurement it is often challenging to isolate a homogeneous sediment sample and obtain the intrinsic frequency dependence. One approach for isolating a homogeneous sample is through mechanical sampling, e.g., a coring device, identifying a uniform region in the cored material and then conducting measurements on that sub-sample. This approach has significant disadvantages: 1) mechanical sampling introduces changes to the sediment, and most especially to fine-grained 
sediments which have a fragile structure that is easily altered, 2) it is usually difficult/impossible to determine what the sampling effects were, and 'undo' them in the analysis and 3) the resulting sub-sample sizes only permit measurements of sound speed and attenuation at high frequencies (order hundreds of kilohertz), since the dimension of the sub-sample is generally small, of order centimeters.

An alternative approach is to use acoustic remote sensing of the sediment geoacoustic properties, and then 'isolate' a homogeneous sample. In order to achieve this, numerous potentially confounding frequency-dependent mechanisms must be separated in order to obtain unbiased intrinsic sound speed and attenuation. These include non-sediment related mechanisms such as effects of scattering from sea surface roughness/bubbles, effects of space/time-dependent ocean dynamics, e.g., internal waves, and scattering from biologics. More subtly, sediment-related structures, if not detected and accounted for, also lead to biases. The structures include sound speed and attenuation gradients due to increasing overburden pressure or smoothly changing porosity (e.g., [3]), discrete layers greater than $\sim \lambda / 8$, and seabed lateral variability. In the New England Mud Patch (NEMP) environment, these are considered to have primary effects on the frequency dependence of sound speed and attenuation. Secondary effects include effects of shear waves and associated gradients; secondary because the shear wave speed in muddy sediments is quite low, a few tens of meters per second [4],[5]. Other possible confounding mechanisms are scattering from interface roughness and from sediment volume inhomogeneities.

Given the mechanisms above that need to be teased apart in order to recover the intrinsic frequency-dependent sound speed and attenuation, additional science questions must be addressed:

1. how do the geoacoustic properties vary vertically? This is needed to isolate and quantify properties of individual homogeneous layers

2. How do the geoacoustic properties vary laterally at decimeter to kilometer scales? This can be divided into:

a. small-scale heterogeneities (sediment volume and interface roughness) which cause scattering

b. larger-scale lateral heterogeneities or geoacoustic range-dependence.

Therefore, in order to compare observations with theory, it is critical that the theoretical basis (wave propagation through a homogeneous sample) is respected as strictly as possible for the observations. Thus, considerable care is required in designing the measurements.

A primary short-term goal for the present work is to address the important question of the sound speed of the mud near the water-sediment interface. This is a deceptively challenging measurement, as indicated by the wide range of sound speed ratio estimates to date, from 0.945 -1.026 over the band $40-1000 \mathrm{~Hz}$, NEMP (Ref [6], Fig. 7) from more than a dozen different measurements, corresponding to mud sound speed disparities up to $\sim 120 \mathrm{~m} / \mathrm{s}$. Thus, at this stage, it is not even clear whether or not the mud has a sound speed less than or greater than that of the water. As another data point, the commonly used Bachman-Hamilton empirical relations [7] using the porosity of the mud, 0.60 [8], yields a sound speed ratio of 1.04, which further increases the possible range of sound speed values. 
In measurements reported here, the data show an unambiguous angle of intromission of $8^{\circ}$. The mere existence of an angle of intromission means that the sound speed of the sediment must be less than that of the water column. We term this 'direct' geoacoustic information - no models or inversion are required. Of course, a theory of wave reflection is required, but the conclusion (sound speed ratio less than 1) is independent of whether the model of the sediment is a fluid, or a viscoelastic solid, or a poro-viscoelastic solid, or whether the wavefield is modeled as a plane wave, or spherical wave. If a model is coupled to the observation, further quantitative information can be had, namely the sound speed ratio.

Despite the considerable value of the angle of intromission for probing in-situ fragile muddy sediments, measurements are exceedingly rare. To the authors' knowledge, there are no published angle of intromission measurements on the continental shelf of the United States, nor yet the entirety of North America. This scarcity may not be because muddy sediments exhibiting an angle of intromission are scarce, but rather because the measurements are challenging. It should also be pointed out that not all muddy sediments are expected to exhibit an angle of intromission. Measurements are required. The angle of intromission measurements at the NEMP presented here provide unique and valuable understanding of the sound speed in the upper few meters of sediment.

The paper is organized as follows. In Section II, the measurement philosophy is further discussed along with the specific experiment designs to address the science questions. In Section III, direct geoacoustic information is presented and discussed, including observations of the angle of intromission and critical angle. In section IV, time domain measurements are presented and discussed in terms of both vertical and lateral geoacoustic properties. Section V summarizes the results.

\section{Measurements}

A common and powerful geoacoustic inference technique is to exploit waveguide physics, where source and receiver are widely separated, kilometers to tens of kilometers in shallow water. One challenge faced by the waveguide methods is that oceanographic variability can cause contaminating effects in the data that are difficult to separate from seabed effects. To obviate this difficulty, the SBCEX17 experiment was specifically executed during winter months when the water column is well-mixed. A remaining oceanographic feature that could cause difficulty for waveguide seabed inference methods is scattering from the sea surface and/or bubble clouds in high sea states and frequencies above a few hundred hertz. Another challenge to the waveguide measurement method is a) that the seabed is variable over kilometer to tens of kilometer lateral scales, b) that widely separated source and receiver observations do not have the geoacoustic information content to recover the seabed lateral variability and c) when the known or unknown lateral variability is ignored, the resulting estimated geoacoustic properties are biased (e.g., [9],[10]). Another challenge is that there is generally insufficient geoacoustic information content in the data to recover the details of the depth-dependent geoacoustic properties of the seabed. This is due in part to sensitivity of the data to the ocean water column, in part to smearing of the information in range, and in part to the low frequencies generally employed. As but one example, the geoacoustic information content in long-range propagation data are insufficient to directly detect the angle of intromission and in fact are relatively insensitive to the sediment sound speed 
ratio near unity. This can be seen from ten widely varying estimates of sound speed ratio, 0.945 -1.026, from $40-1000 \mathrm{~Hz}$ long-range propagation data sets (Ref [6], Fig. 7 which includes only sound speed ratio estimates at/near the water-mud interface).

In summary, it is difficult using long-range acoustic data to isolate a homogeneous portion of the seabed from all other mechanisms which is needed for measuring frequency-dependent sound speed and attenuation.

To reduce these difficulties, experiments were designed/conducted with short source-receiver offsets. That is, instead of using waveguide physics, we exploit the physics of a single interaction with the seabed. Typically, the insonified lateral region was of order ten to a hundred meters. The short distance of the path through the water column and small seabed footprint greatly reduces the contaminating effects described above; in consequence, the sensitivity of the measured signal to the geoacoustic properties is amplified. The sensitivity to geoacoustic properties is further enhanced by probing the seabed not only at sub-critical, but at post-critical grazing angles where the reflected pressure contains greater geoacoustic information layer by layer, especially attenuation. Generally, geoacoustic information content increases with increased angular range and with increased frequency range (bandwidth). This is true for both reflection and scattering data. At very low grazing angles, neither the scattering cross-section (which approaches zero) nor the reflection coefficient (which approaches unity) has significant information content. Since theory shows that the seabed scattering cross-section is a strong function of the seabed reflection coefficient, the experiment design sought to jointly maximize geoacoustic information content for reflection and scattering measurements.

The experiments were designed to address the two science questions (listed in Section I) with two kinds of experiment geometries. To address sediment vertical variability, a broadband source is towed in radials centered at a bottom-moored receiver, Figure 1a. Data from this experiment yield the reflection coefficient as a function of angle and frequency; analysis yields the vertical, or depth-dependent geoacoustic properties laterally averaged over about a water depth. To obtain sediment lateral variability, a source and a receive array are towed near the seabed, see Figure $2 \mathrm{a}$. Analysis of these data yield: a) the depth- and lateral-dependent geoacoustic properties of the seabed with a resolution of about $0.5 \mathrm{~m}$ and $10 \mathrm{~m}$ respectively over a track extending tens of kilometers and b) roughness spectra at various interfaces and sediment volume heterogeneity spectra. For ease of reference these two experiments will be denoted as 1-D (aimed at determining the depth-dependent geoacoustic properties) and 2-D (aimed at depth- and laterallydependent geoacoustic properties). 
a)

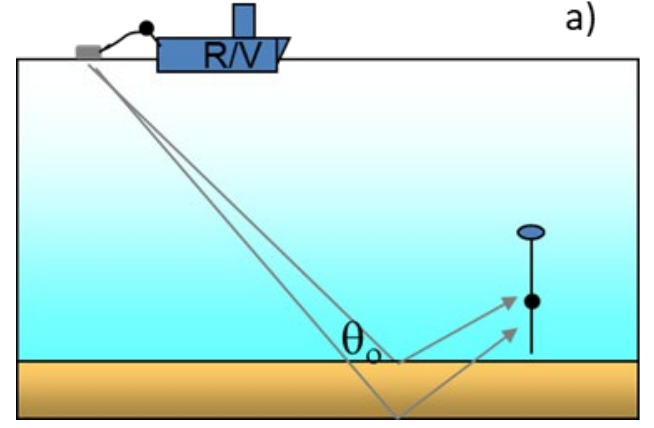

b)

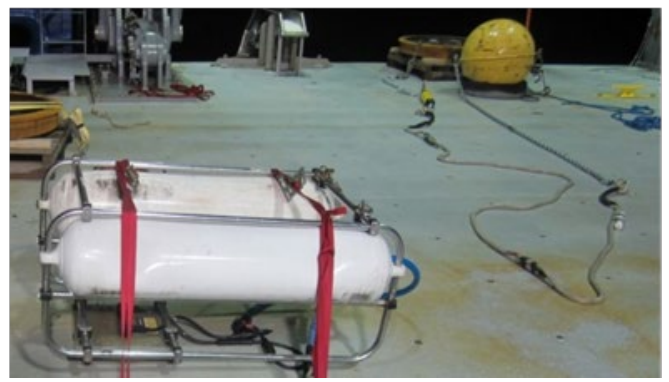

Figure 1. a) Wide-angle seabed reflection experiment cartoon showing the broadband source towed behind the research vessel and the bottom-moored receive hydrophone string and $b$ ) the source catamaran (foreground) with source plate (behind the red strap on the left) and the receive mooring with yellow float next to the ballast (train wheel) and hydrophones attached to the mooring rope.

a)

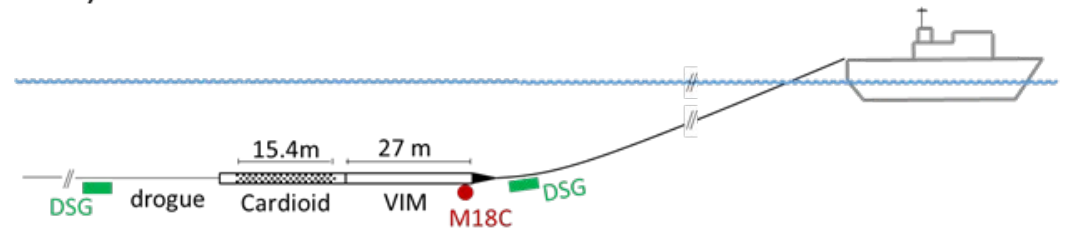

seabed b)

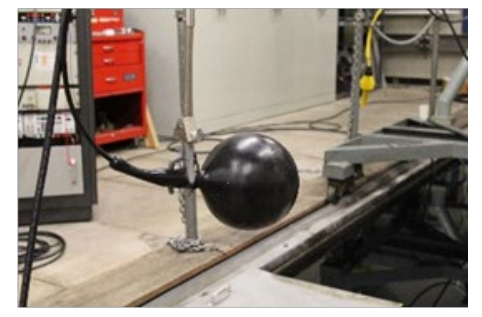

Figure 2. a) cartoon of seabed interaction experiment with a towed M18C source and cardioid receive array. The moving-source moving-receiver experiment yields data from which 2-D geoacoustic properties (depth in the seabed and offset along a transect) can be obtained as well as scattering measurements. The distance from source to first cardioid triplet is $30 \mathrm{~m}$. Two selfrecording hydrophones (DSG) were mounted to the cable and drogue $\sim 5$ and $112 \mathrm{~m}$ respectively from the source. b) photo of the M18C 6-inch diameter source in the lab prior to calibration.

The locations of the experimental sites were chosen collaboratively with other scientists from the SBCEX community (e.g., see [11]), such that multiple observational methods and inference techniques could be brought to bear at the same locations. Figure 3 shows the experiment area on the New England Mud Patch along with the 1-D experiment sites (green and cyan circles) which sample various mud thicknesses. At the VC31-2 site the mud was only a few meters thick, whereas at the SWAMI site near the thickest region of the Mud Patch, the mud thickness was $\sim 10$ $\mathrm{m}$. The former site is named for its core designator, while the latter site was named for proximity of the ARL-UT SWAMI array (though the ARL-UT SWAMI array was not used as a receiver for the reflection measurements). Thus, the term 'SWAMI site' used in this paper always refers to the reflection measurement location $\sim 1 \mathrm{~km}$ WNW of the ARL-UT SWAMI array. The site in the north-east corner used a 64-hydrophone $1 \mathrm{~km}$ long horizontal array (FFI, Norway) on the seabed as the receiver. Two traverses were performed, along (East-West) and perpendicular (NorthSouth) to the FFI array. The mud thickness at the FFI site was $\sim 5 \mathrm{~m}$. 
Along the 2-D experiment tracks, two equalized Linear Frequency Modulated (LFM) $0.25 \mathrm{~s}$ down-sweep pulses were broadcast, from 6 to $1 \mathrm{kHz}$ and 6 to $0.5 \mathrm{kHz}$. Each pulse type had a $1 \mathrm{~Hz}$ repetition rate and the two pulses were interleaved by $0.5 \mathrm{~s}$. The tracks are shown in Figure 3. Both the March 30 'racetrack' and the April 3 designs were motivated by the objectives

- to measure 2-D geoacoustic properties and small-scale sediment heterogeneity over large $(\sim 30 \mathrm{~km})$ spatial distances and over widely varying mud thicknesses (from $\sim 3$ to $12 \mathrm{~m}$ ). The two east-west $\sim 28 \mathrm{~km}$ (March 30 ) tracks were separated by $1 \mathrm{~km}$ - another sediment heterogeneity scale to probe.

- to collect 2-D and 1-D data at the same site. This was accomplished at both the SWAMI and SC2 sites; the data sets at the same site provide an opportunity for inter-comparison of 2-D geoacoustic results with those from the 1-D data. The latter are expected to contain higher geoacoustic information content and lower uncertainties due to a wider angular range

- to conduct 2-D measurements over sites/tracks planned by other researchers for intercomparison. For example, numerous long-range acoustic propagation experiments were conducted by other researchers along the $\mathrm{x}$-shaped pattern of cores (magenta points) in the central area of the experiment box, see Figure 3 . The crossing point of the ' $x$ ' is near the thickest point of the mud layer, $\sim 12 \mathrm{~m}$. The April 3 lines (blue) traverse along the same bearings as the cores and other researchers' experiments; designed to coincide with the propagation tracks and provide higher lateral/vertical resolution geoacoustic properties than possible from long-range propagation data.

- to examine 2-D data repeatability by conducting the same experiment along the same track multiple times. This was partially accomplished on March 30 (red line); mechanical issues prevented the planned acquisition start at the western end of the northern leg. Instead, acquisition began at $\sim 70.65^{\circ} \mathrm{W}$ on the northern leg, transiting east. Shortly after the first full racetrack was completed, another research vessel occupied the track, which necessitated an unplanned turn to the south-east, see Figure 3. This left a short, $\sim 900 \mathrm{~m}$, track section starting at $\sim 70.65^{\circ} \mathrm{W}$ from which raw data and inferred geoacoustic properties can be compared along the same track. The April 3 track has multiple passes near the center of the X-coring pattern.

- To examine azimuthal variability. The multiple crossing points along the March 30 and April 3 tracks at the SWAMI site were intended to permit inter-comparison of the 2-D data along different bearings.

Marine mammal activity in the area on April 3 limited transmissions, which resulted in no data collected along the southwestern leg of the coring pattern.

In addition to the towed array receiver on April 3, a short bottom-moored hydrophone string was deployed near the former position of Scripps 2 array along the south-eastern leg (cyan circle in Figure 3). The moored hydrophones collected valuable data from the towed source transmissions including: wide-angle 1-D reflection data, long range propagation data and a check on the $\mathrm{M} 18 \mathrm{C}$ source tank calibration with the source mounted on the towed array vibration isolation module (VIM) at a depth of 50-60 m. 


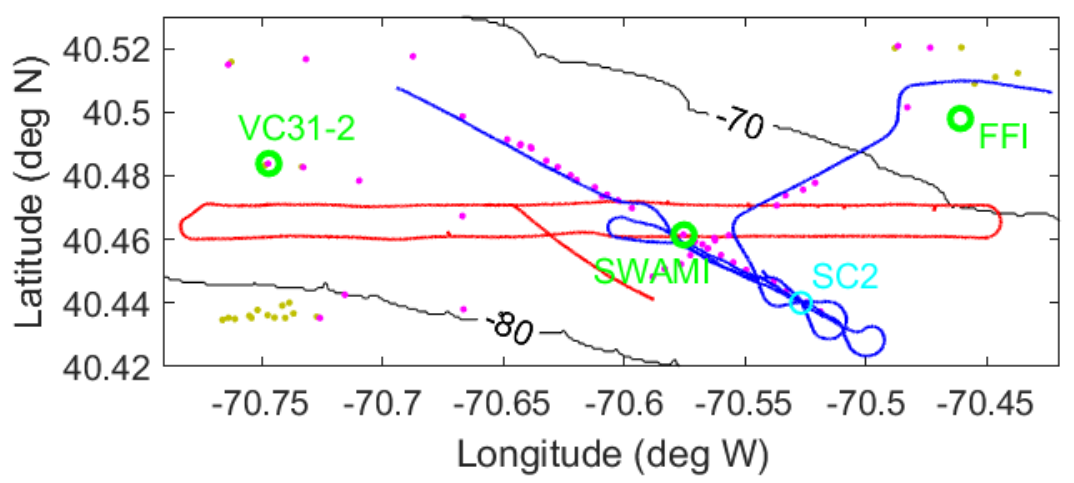

Figure 3. Map of experiment area with bathymetry $(m)$, cores (dots), wide-angle reflection (1-D) measurements with a fixed receiver (4 circles) and source-towed array (2-D) tracks March 30, 2017 (red) and April 3, 2017 (blue). The 1-D measurements were performed with a broadband incoherent source (green) and a M18-C coherent source (cyan). The receiver for the VC31-2, SWAMI and SC2 sites was a short bottom-moored hydrophone string and for the FFI site (green) it was the FFI array.

In the following sections, the experiment hardware and sensors are described in more detail for the two measurement geometries. New experiment geometries, i.e., not described in prior publications, are described in greater detail.

\section{A. 1-D reflection measurement design}

\section{Receivers}

Two different receivers were employed. At the FFI Site, the receiver was the FFI horizontal line array. At the three other sites (SWAMI,VC31-2, and SC2) the receiver was a bottom-moored sparse vertical array with two icListen hydrophones, a Loggerhead DSG hydrophone, an RBR temperature and depth recorder (TPod), and an Ultra-Short-Baseline (USB) transponder. The buoyancy member was a 28 inch steel sphere. At the SWAMI and VC31-2 sites the two icListen hydrophones, DSG, RBR, USB, and sphere were nominally 11.5, 15.8, 20, 25, 27, and $31 \mathrm{~m}$ above the seabed respectively. At the SC2 site, the sensors were closer to the seabed at 4,8,10,11 and $15 \mathrm{~m}$ in order to reduce entanglement risk with the towed array. The bottom-moored array was stable on each deployment, with TPod peak-to-peak depth oscillations $\sim \pm 0.05 \mathrm{~m}$ on March 28 and March 31 over a broad period of $9-15 \mathrm{~s}$, and an additional narrow peak period $\sim 17 \mathrm{~s}$ on March 28. On April 3, 2017 depth oscillations were $\sim \pm 0.1 \mathrm{~m}$ with a period of $11-15 \mathrm{~s}$. The icListen hydrophone dynamic range is $118 \mathrm{~dB}$ which eliminates the need for gain changes at various ranges (as required in early versions of this experiment geometry [12]). The icListen sampling frequency was $32 \mathrm{kHz}$, except on March 28, 2017 (VC31-2) when the phone closest to the seabed was $16 \mathrm{kHz}$. Only data from the upper icListen hydrophone is presented here.

Following deployment, receive array locations were determined using the USB system, which involved crossing over the estimated array location multiple times. The receiver locations from the USB system on March 28, March 31, and April 3, 2017 are 70.7469 W 40.4838 N, 70.5753 W $40.4614 \mathrm{~N}$ and $70.5269 \mathrm{~W} 40.4401 \mathrm{~N}$ respectively. The radial crossing point over the FFI array was $70.461 \mathrm{~W} 40.498 \mathrm{~N}$.

\section{Sources}


Two sources were employed. At three sites, the source was an Applied Acoustics AA201 boomer plate (Figure 1b) mounted on a $1 \times 2 \mathrm{~m}$ catamaran at a depth of $\sim 0.4 \mathrm{~m}$. The AA201 plate is 19 $\mathrm{cm}$ in diameter and emits a short, $\sim 0.3 \mathrm{~ms}$, highly repeatable pulse every second with a useable band from a few hundred hertz to a few kilohertz. In the first deployment, the catamaran was deployed from the starboard side crane and towed via the source electrical cable. The initial test showed the catamaran pitching forward under tow. To improve balance, the plate was moved to the aft end of catamaran, see Figure 1b, where the tow point is to the right. Moving the source plate improved the tow characteristics. A second issue was that the catamaran tended to set just inside the ship wake. The concern of bubbles contaminating the source signature prompted shortening the tow/source cable, so that the catamaran moved forward out of the wake. The first reflection experiment, March 28, 2017 (VC31-2 and FFI) was conducted with this setup. One of the remaining problems was that the catamaran was fairly close to the ship hull, $\sim 5 \mathrm{~m}$, and there was concern for potential contamination by hull reflections. In the subsequent deployment, March 31, 2017 (SWAMI) the tow point was moved to the block under the A-frame and the catamaran was towed by a separate tow line, instead of the source cable. Also, the source power unit was shifted from the lab to the aft hangar which allowed sufficient source cable for the catamaran to be towed $\sim 20 \mathrm{~m}$ behind the stern where wake bubbles did not appear to affect the result and the catamaran appeared to ride more stably than on the March 28 experiment.

The second source was a GeoSpectrum M18C-6 $15.5 \mathrm{~cm}$ spherical (omnidirectional, see Appendix A) transducer with a resonance frequency of $12.4 \mathrm{kHz}$. This source was used at site SC2. Equalized Linear Frequency Modulated (LFM) 0.25 s downsweep pulses were broadcast, from 6 to $1 \mathrm{kHz}$ and 6 to $0.5 \mathrm{kHz}$. The latter pulses were significantly lower in amplitude due to the amplitude equalization.

\section{B. 2-D reflection and scattering measurement design}

The towed receiver was the $15.4 \mathrm{~m}$ aperture cardioid module from the ONR Five Octave Research Array at Penn State, FORA [13]. From a science standpoint, one of the longer FORA modules (either the $23.6 \mathrm{~m}$ or $47.2 \mathrm{~m}$ aperture) would have been preferable over the cardioid, to measure a wider range of seabed reflection angles (hence increased geoacoustic information content). However, the longer aperture modules do not record individual phones, but rather phone groups and thus were not suitable for individual phone processing needed for the reflection analysis. The cardioid module is oil filled, $88 \mathrm{~mm}$ in diameter and contains 234 hydrophones arranged in 78 hydrophone triplets with a $12.5 \mathrm{kHz}$ sampling frequency. Each triplet set consists of three hydrophones on an equilateral triangle with $38.5 \mathrm{~mm}$ spacing between the individual phones in a plane perpendicular to the array axis. The 78 triplet sets are spaced along the array at $0.20 \mathrm{~m}$. The true dynamic range after accounting for electrical and quantization noise is $\sim 106 \mathrm{~dB}$. In addition to the acoustic sensors, the array has a non-acoustic sensor (NAS) suite $2.62 \mathrm{~m}$ forward of the first hydrophone which provides heading, pitch, roll, and a temperature and a depth sensor placed $1.25 \mathrm{~m}$ further forward. A depth and temperature sensor is also located $4.48 \mathrm{~m}$ aft of the last hydrophone. The receiver was towed at depths of 50-65 m.

The source, a GeoSpectrum M18C-6, was selected because its transmit beampattern is virtually omnidirectional over the frequency range of interest (see Appendix A). A source with a strong beampattern is problematic since the reflection coefficient and scattering cross-section are quite sensitive to the beam pattern and the concomitant required precise $3 \mathrm{D}$ positioning under tow is 
technically challenging. The challenge with the M18C-6 is to tow a $15.5 \mathrm{~cm}$ diameter sphere in a stable fashion. Mounting it under a tow fish was considered, however reflection and scattering from the towfish structure would have a deleterious effect on the seabed reflection and scattering data quality. Furthermore, the source (towfish) to receiver array separation would vary from ping to ping throughout the experiment evolution. To obviate these issues, the source was mounted directly to the VIM (which seemed a more stable arrangement than mounting on the cable). The mount was implemented with a minimum of hardware in order to avoid altering the source's omnidirectional characteristics. The mount consisted of a strong nylon mesh wrapped several times around the M18 source and array VIM; heavy-duty cable ties were woven through the mesh fore and aft of the source and tightened around the VIM securing the source and source cable. This necessitated tie-wrapping the source cable to the FORA power/tow cable on each deployment which was rather labor and time-intensive. Nevertheless, the array with the source attached appeared to tow quite stably.

The source was placed in the VIM at the maximum distance from the first hydrophone, $30 \mathrm{~m}$, in order to probe the lowest possible grazing angles (though all post-critical). Since the seabed reflection coefficient varies more rapidly at low than steep grazing angles, the lower grazing angles provide higher geoacoustic information content. In order to obtain a wider range of reflection angles than possible from the cardioid array, two additional self-recording hydrophones (DSG) were attached to the tow cable/drogue at 4.75/112 $\mathrm{m}$ forward/aft of the source, see Figure 2a. At a $15 \mathrm{~m}$ source-receiver altitude above the seabed, bottom grazing angles from the cardioid module are $33-45^{\circ}$ and from the two DSGs, $81^{\circ}$ and $15^{\circ}$. A second purpose of the DSG closest to the source was to measure quasi-monostatic seabed scattering. On the April 3, 2017 deployment, the DSG attached to the drogue (112 $\mathrm{m}$ from the source) was dislodged and lost.

The water column properties were measured with a conductivity-temperate-depth probe immediately before and after the measurements. Consistent with extensive ocean measurements (including moorings) from other researchers [6], the sound speed profile was nearly isothermal, and changed little over the course of the experiments.

\section{Seabed reflection observations, 1-D}

There is significant geoacoustic information content in the seabed reflection data both in the time domain and in the frequency domain. Many different methods have been developed to access that information. Broadly, the methods can be divided into 'direct', i.e., methods that provide geoacoustic information without inversion and 'indirect', i.e., methods that require inversion to obtain the geoacoustic properties. In the following we present the data in the time and frequency domains and the direct geoacoustic information content. Another analysis of the reflection measurements using indirect methods is found in [14].

\section{A. Time domain analysis}

An example of raw time series data from a single ping is shown in Figure 4 where the direct path and reflection from the various interfaces can be seen, including the water-mud interface, the sand-mud interface and the sea surface. It is even easier to see the arrivals over multiple pings, Figure 5a, where the arrivals in slow (UTC) time nearly follow hyperbolae. In particular, the mud-sand horizon is easier to observe than in Figure 4. A still clearer presentation of the arrivals is shown in Figure $5 \mathrm{~b}$ where data are mapped in reduced time $t_{\text {red, }}$, 


$$
t_{\text {red }}^{2}=t_{o}^{2}-\left(\frac{r}{v}\right)^{2}
$$

where $r$ is source-receiver offset, $t_{o}$ is the arrival time at $r=0$, and $v$ is the reducing velocity. This mapping removes the hyperbolic trend from the data. That is, arrivals in reduced time are approximately independent of source-receiver offset when the $v$ is close to the root-mean-square (rms) sound speed. Here $v=1473 \mathrm{~m} / \mathrm{s}$, the water column sound speed. Thus, the direct path and water-mud arrival reduced time are nearly independent of offset.

The salient features to note at this point are the amplitudes of the water-mud and mud-sand arrivals versus offset. The water-mud arrivals are strongest near normal incidence (zero offset) then decay with offset. This behavior is consistent with a sediment whose sound speed is less than that of the seawater, where the amplitude decreases from normal incidence to near zero at the angle of intromission, see Figure 6a. It is not easy to detect if the angle of intromission occurs in the raw data, Figure $5 \mathrm{~b}$, but the evolution of amplitude does show that the sediment sound speed is close to that of water. The mud-sand horizon amplitude shows more or less the opposite behavior, the reflection amplitude is small near normal incidence and then shows an abrupt increase at about $160 \mathrm{~m}$ offset. This abrupt increase is due to the critical angle, see Figure $6 \mathrm{~b}$. Thus, by simple inspection it is clear that the lower horizon (sand) sound speed is considerably higher than that of the mud.

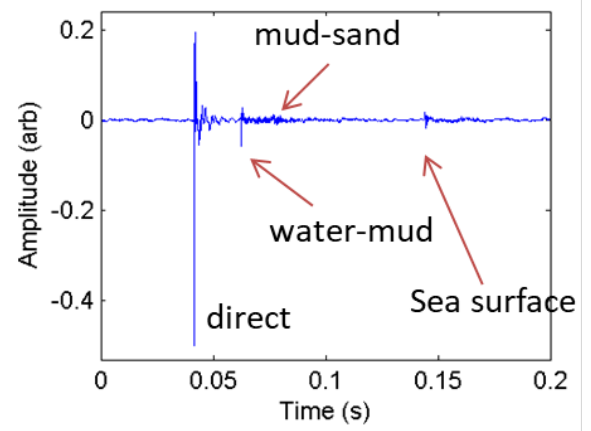

Figure 4. raw time series at SWAMI site for a single ping near the closest point of approach.
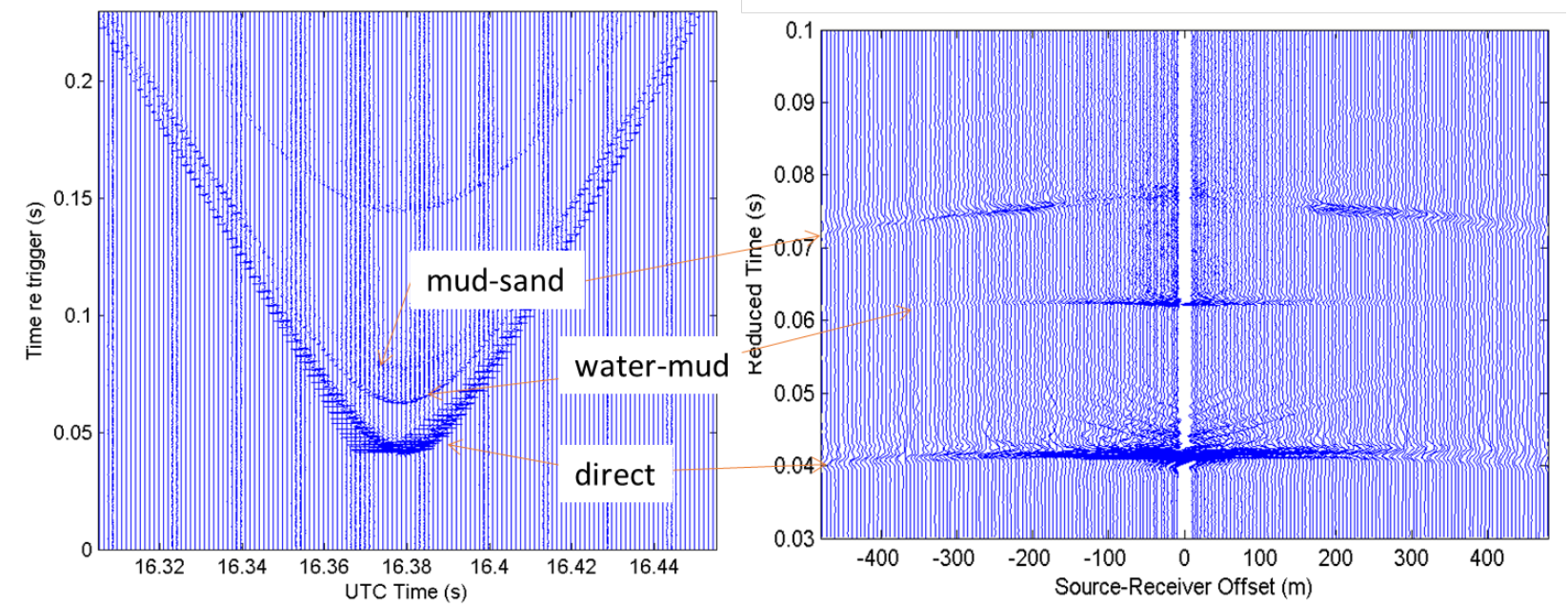

Figure 5. stacked raw time series at SWAMI site a) in linear time and b) in reduced time. Note that between the direct arrival and the reflection from the water-mud interface there is low-frequency ringing (coda) from the source. 

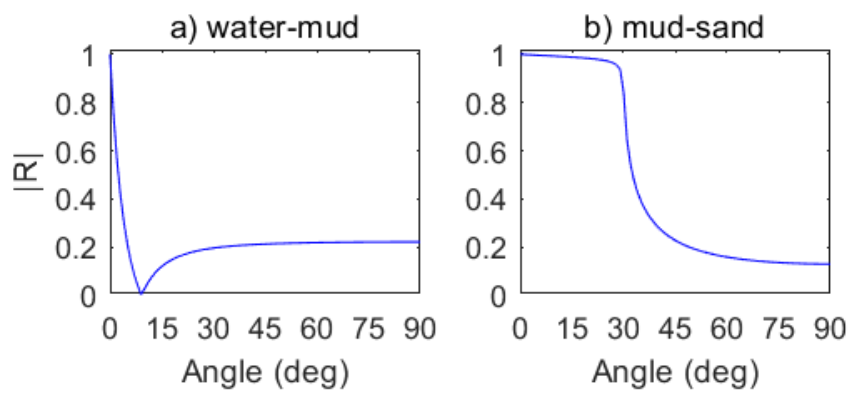

Figure 6. hypothetical reflection coefficients at a) water-mud and b) mud-sand boundaries. The mud-sand normal incidence reflection coefficient is smaller than that of the water-mud reflection primarily because the density ratio is smaller.

In seismology, geoacoustic inversion is widely performed from the time domain response, that is by picking peak amplitudes of individual layer horizons. That observable and its forward model, however, requires the implicit assumption that the reflection horizon is fully resolved by the pulse, i.e., that the reflected field can be properly represented by two homogeneous halfspaces in contact. Given the wide range of seabed scales and processes, this assumption can be invalid in many situations. Moreover, it is not generally possible to determine when the underlying assumption is or is not valid. Yet another disadvantage is the need to determine the layer peak amplitude. While at first blush it may seem straightforward to pick a peak, factors such as the sampling frequency and the signal-to-noise ratio can make peak amplitude estimation subjective.

Instead, the observable chosen for this research is the frequency-domain seabed reflection coefficient. It does not require any assumptions about the boundaries between layers, nor does the peak amplitude need to be estimated. Most to the point, the geoacoustic properties, sound speed, density and especially attenuation, are generally better estimated in the frequency domain rather than time domain.

\section{B. Frequency domain analysis \\ 1. Theory}

We begin with a discussion about the measurement quantity. The simplest quantity that describes acoustic interaction with the seabed is the plane wave pressure reflection coefficient, $R_{p}$ defined as the ratio of the reflected to the incident pressure at a specific angle. However, experimentally a spectrum of angles is generated, not a single plane wave. Thus, for a given source-receiver offset the reflected field contains a spectrum of angles, i.e., not just the specular angle at the seabed. This is so because reflection occurs over a finite region at a boundary, the Fresnel zone, that includes a spectrum of incident and reflected angles. Further, the seabed is generally a layered medium and each layer will have distinct specular angle and angular spectrum, see Figure 1a, which shows the specular angle difference at two interfaces. The full physics solution for the reflected field at the receiver is the sum of reflected waves at all angles and is given by the Sommerfeld-Weyl integral, here cast in the angular rather than in the usual wavenumber domain

$$
p_{r}\left(\theta_{o}, f, z_{t}\right)=i k \int_{0}^{\pi / 2-i \infty} R_{p}(\theta, f) J_{0}(k r \cos \theta) e^{-i k z_{t} \sin \theta} \cos \theta d \theta
$$


where $\theta$ is grazing angle in the vertical plane; $\theta_{o}$ is the specular angle $\theta_{o}=\tan ^{-1}\left(z_{t} / r\right) ; J_{0}$ is the Bessel function of order $0, k$ is the wavenumber in the water, $f$ is frequency and $z_{t}$ the sum of the source and receiver heights. Note that $R_{p}$ is completely general and represents any arbitrary planelayered medium. The integral thus includes reflections from all sub-bottom interfaces as well as refracted arrivals. It also treats inhomogeneous waves including interface and lateral waves and properly accounts for the Fresnel zone at each interface.

In order to form a useful measurement quantity, a spherical reflection coefficient, $R_{s}$, is defined by scaling $\mathrm{Eq}(2)$ by a unit source, $p_{d}=1$, times the Green's function along the specular path with $R_{p}=1$,

$$
\begin{gathered}
R_{s}\left(\theta_{o}, f, z_{t}\right)=\frac{p_{r}\left(\theta_{o}, f, z_{t}\right)}{p_{i}\left(\theta_{o}, f, z_{t}\right)} ; \quad p_{i}=p_{d} \frac{e^{i k D}}{i k D} \\
R_{s}\left(\theta_{o}, f, z_{t}\right)=\frac{i k D}{e^{i k D}} \int_{0}^{\pi / 2-i \infty} R_{p}(\theta, f) J_{0}(k r \cos \theta) e^{-i k z_{t} \sin \theta} \cos \theta d \theta
\end{gathered}
$$

where $D=\operatorname{sqrt}\left(r^{2}+z_{t}^{2}\right)$. The independent variable $z_{t}$ is explicitly shown as a reminder that $R_{S}$ (unlike $R_{p}$ ) depends upon the experiment geometry. It should be noted that since $R_{s}$ includes a spectrum of angles and arrival paths, energy conservation is not violated for $\left|R_{S}\right|$ greater than unity.

\section{Data Processing}

The data processing follows directly from the theory. Dropping the subscript $o$ for the specular angle for the remainder of the paper,

$$
\left|\hat{R}_{s}\left(\theta, f, z_{t}\right)\right|=\left|\frac{\hat{p}_{r}\left(\theta, f, \tau ; z_{t}\right)}{\hat{p}_{d}\left(\theta_{d}, f, \tau\right)}\right| \frac{\gamma_{d}}{\gamma_{r}}
$$

where $\hat{p}$ is the Fourier transform of the windowed seabed reflected time series, $\gamma$ is the Green's function from source to receiver which includes spreading and absorption calculated via ray theory; subscripts $d$ and $r$ identify the path type: direct and seabed reflected respectively; $\theta_{d}$ is the grazing angle at the source for the direct path to the receiver and $\tau$ is the integration time of the time series. In this work, the integration time starts just before the first seabed reflected return. There are some fine points related to the processing when the source is not omni-directional that for conciseness are not discussed here but are given in [15]. For the incoherent source, it has been found that averaging the data over proportional bandwidths is a good compromise between high geoacoustic information content and noise. For example, reflection data at the SWAMI and VC31-2 sites were frequency averaged over a 1/7.5 octave bandwidth for trans-dimensional inversion [14].

For one of the reflection experiments, site SC2, the source projected a Linear Frequency Modulated (LFM) pulse. In order to properly perform match filtering, Doppler compensation was applied. The relative motion of the source and receiver imparts both a frequency shift and a pulse duration change. The appropriate Doppler compensation was estimated using a brute force search. Finally, note that the normalization in Eq. (5) means that uncertainties in source, receiver and data acquisition system calibrations play a negligible role. The Green's functions are calculated with 
the measured sound speed profiles, which were nearly isothermal and stable throughout these experiments.

When it is possible to synchronize source and receiver clocks, obtaining accurate source-receiver offsets and depth is straightforward using observed-versus-modeled direct path arrival times. When the clocks are not synchronized, as is the case in this experiment, the localization is performed using a linearized Bayesian approach [16].

Processing the direct path time series yields the source amplitude as a function of source angle and frequency. An example of measured source levels at two sites are shown in Figure 7, where the main features of the beampattern are quite similar, as expected. Some differences are due to differences in the catamaran towing/source plate configuration. The large variation in the beampattern above $\sim 1.5 \mathrm{kHz}$ led to low quality reflection data since the seabed reflected path signal-to-noise ratio (SNR) was often poor. This source beampattern feature is due to the shallow source depth. The right-most plot shows the theoretical Lloyd mirror pattern for a source depth of $0.4 \mathrm{~m}$, where the source plate was modeled as a point source. The first null near $2 \mathrm{kHz}$ matches reasonably well with the measurements confirming the cause of the variable beampattern. The second and perhaps the third null are also visible in the measured data.
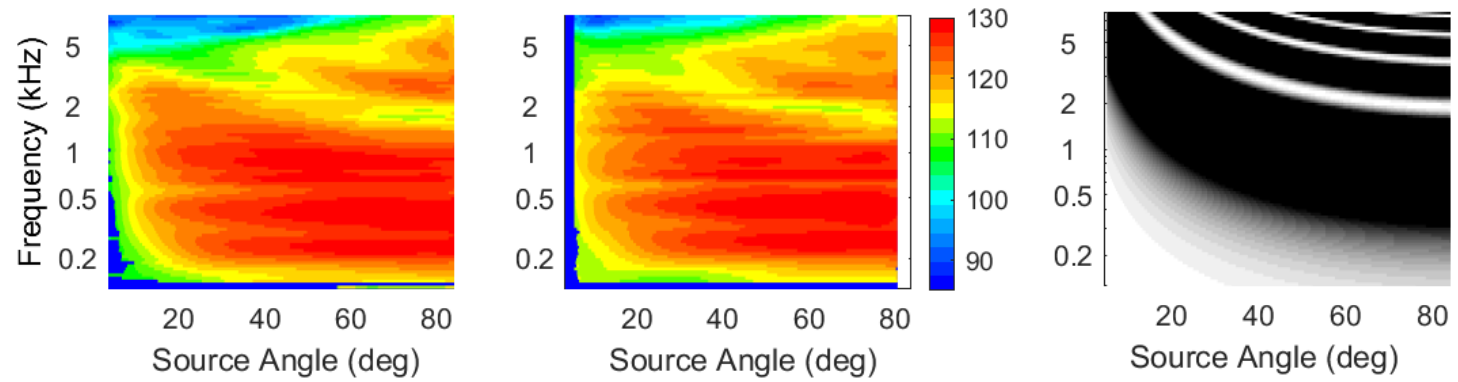

Figure 7. source level (dB re 1uPa ${ }^{2} \mathrm{~s} / \mathrm{Hz} @ 1 \mathrm{~m}$ ) for VC31-2 (left) and SWAMI (middle), Lloyd mirror pattern, white is a null. Note the presence of the nulls in the measured source beampattern.

The final step in the data processing is windowing the seabed reflected path, transforming the data to the frequency domain and then forming the reflection coefficient. The minimum signal-tonoise ratio used in the data processing is $6 \mathrm{~dB}$.

\section{Results for a long integration time}

The integration time, $\tau$, provides a means for further increasing the geoacoustic information content of the measured data. For example, consider the time series in Figure $5 \mathrm{~b}$ from the SWAMI site. In order to determine the depth dependence of the mud and sand layers, the reflection coefficient is computed from a window that begins just above the water-mud interface and ends a few meters below the upper sand interface(s) at a depth of $16 \mathrm{~m}$ sub-bottom. The resulting reflection coefficients are shown in Figure 8a. The most obvious feature is the clear critical angle at $\sim 27^{\circ}$ which can provide an initial estimate of the sandy layer(s) sound speed below the mud. 
Another apparent feature is the Bragg interference pattern. Bragg's law gives the condition for constructive interference and can be written for a sediment layer $j$, as $k_{j} d_{j} \sin \left(\theta_{j}\right)=n \pi$, where $k$ is the wavenumber, $d$ is layer thickness, $\theta$ is grazing angle, and $n$ is an integer. The pattern of alternating constructive and destructive interference can be seen at all angles below $\sim 1500 \mathrm{~Hz}$. The primary interference comes from waves reflecting off the water-mud and mud-sand interfaces. Other interference patterns from thinner layers are less easy to detect in this image. The Bragg interference contains information about the thickness, sound speed, density, and attenuation of each layer through 1) the evolution of the Bragg lines across angle and frequency, 2) the interference peak-to-trough amplitudes, and 3) the decay of the peak-to-trough amplitude as a function of frequency. The first and last of these data features contain significant information about the frequency dependence of the sound speed and attenuation in each layer respectively. Some of this geoacoustic information can be accessed through statistical inference, e.g., [14] and some via forward modeling.

The presence and stability of the Bragg interference in the data are an indication that the sediment is plane-layered (an important assumption in our theoretical model) and that the processing assumptions are met. Some of the Bragg lines below the critical angle, e.g., $\sim 20^{\circ}$ are smeared across frequency. This has not been observed in reflection data to date, despite data being collected in a wide variety of sedimentary environments in the Mediterranean Sea. The cause of the smearing was found to be due to high-speed layers, greater than roughly $2000 \mathrm{~m} / \mathrm{s}$, with relatively low attenuation. These layers are below $16 \mathrm{~m}$ sub-bottom, i.e., below the mud layer and the upper sand layers, and thus do not appear in the time window for the majority of the offsets/angles. However, because of their high sound speed, their arrivals cross over the mud-sand arrivals at far offsets (low angles) and contaminate the reflection processing results at some low angles. Their presence is subtle and not easily seen in the raw data, Figure 5b. It may be possible with signal processing to remove or reduce the effect of these arrivals, however this has not been attempted at this stage.
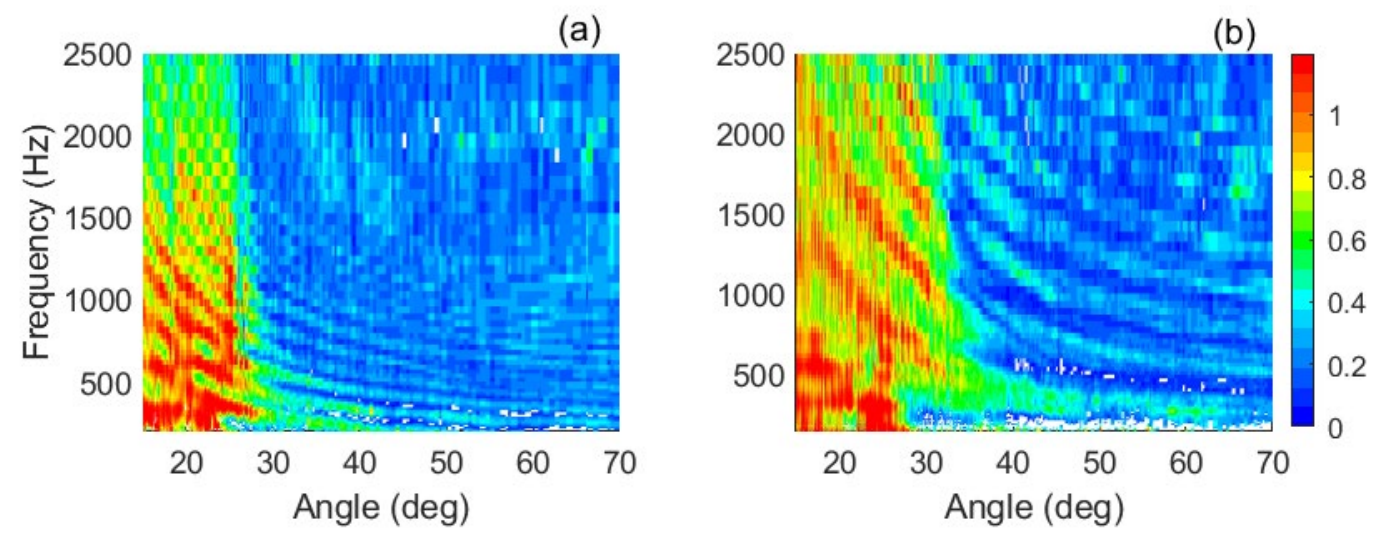

Figure 8. reflection coefficient data from the a) thick mud (SWAMI) site where the integration window is $16 \mathrm{~m}$ and $b$ ) thin mud (VC31-2) site where the integration window is $14 \mathrm{~m}$. The fringe patterns are from Bragg interference, i.e., the coherent interaction of waves reflected various layer horizons. No angular smoothing has been applied.

Processed spherical reflection coefficient data at site VC31-2 is shown in Figure 8b. Comparing this with the SWAMI data, Figure 8a, a number of similarities and differences can be observed. First, both sites exhibit clear Bragg interference patterns and at both sites the interference comes 
from the water-mud and mud-sand boundary reflected waves. Since the VC31-2 mud thickness is $\sim 3.5$ times smaller than that at SWAMI, the peaks and nulls at VC31-2 are spaced further apart by a factor of $\sim 3.5$. Note also the clear critical angle at both sites. The fact that the critical angle is somewhat larger at VC31-2, indicates that there are higher sound speed layers than at the SWAMI site in the upper 14-16 $\mathrm{m}$ of the sediment column.

The angular resolution of the reflection coefficient is a function of the ship speed, typically $\sim 2$ $\mathrm{m} / \mathrm{s}$, the pulse repetition rate $1 \mathrm{~s}$, and the geometry. The angular resolution is $\sim 1^{\circ}$ at $60^{\circ}$ and $0.1^{\circ}$ at $15^{\circ}$ at the SWAMI and VC31-2 sites, see Figure 9 (solid line). For the M18C source towed at $\sim 15 \mathrm{~m}$ above the bottom to the bottom-moored hydrophone, the angular resolution is larger by about a factor of 4 (dash dot). The factor of 4 comes from the fact that $z_{t}$ was about a factor of 4 smaller for the M18C source (near the bottom). Angular averaging can be applied for several reasons - it reduces the amount of non-independent data (i.e., very closely spaced angles do not carry any additional geoacoustic information), it reduces computational cost in statistical inference, and it reduces noise. As can be seen from the pixel size, the data in Figure 8 have no angle smoothing.

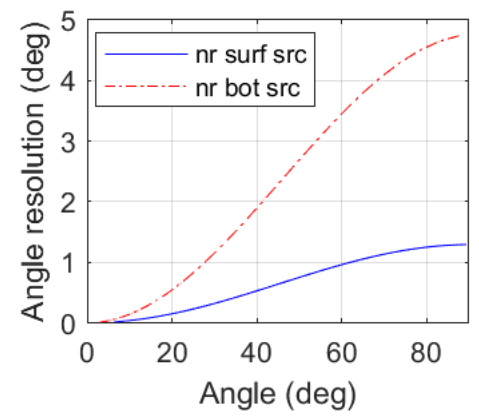

Figure 9. angular resolution of the reflection coefficient data from the near surface source (solid) at the SWAMI and VC31-2 sites, and the near bottom source at site SC2 (dash dot).

4. Results for a short integration time, the angle of intromission

The angle of intromission is the angle at which the reflection coefficient goes to zero. For marine sediments, it occurs under the conditions that sound speed ratio $\left(\mathrm{c}_{2} / \mathrm{c}_{1}\right)<1$ and density ratio $\left(\rho_{2} / \rho_{1}\right)>1$. The angle of intromission, $\delta$, was derived by Lord Rayleigh [17] as

$$
\cos \delta=\left(1-\left(\rho_{1} c_{1} / \rho_{2} c_{2}\right)^{2}\right)^{1 / 2}\left(1-\left(\rho_{1} / \rho_{2}\right)^{2}\right)^{-1 / 2}
$$

Where the subscripts 1 and 2 indicate the water and sediment respectively. It was shown in [18] that by measuring the reflection coefficient versus angle, picking off the angle of intromission, $\delta$, and the normal incidence pressure reflection coefficient $v$, the sediment density and sound speed can unambiguously be obtained from

$$
\begin{aligned}
& \rho_{2}=\rho_{1}\left(1-4 v /(\cos \delta(1+v))^{2}\right)^{-1 / 2} \\
& c_{2}=\rho_{1} c_{1} / \rho_{2}(1+v) /(1-v)
\end{aligned}
$$

assuming that the water column $\rho_{l}$ and $c_{l}$ are known. The equations can easily be modified if the measurements do not extend to normal incidence. 
In principle, detecting the angle of intromission is a powerful way to probe fine-grained (muddy) sediments, which typically have a relative high porosity and are very fragile. Invasive techniques such as coring and insertion of probes tend to disrupt the tenuous bonds that form the sediment matrix. Thus, a remote sensing method is of particular value. Despite the benefits, relatively few measurements of the angle of intromission exist. This is mainly because the measurements are challenging.

In practice, measuring the absence of something (in this case the absence of a reflected wave) is difficult because it requires a high signal to noise ratio; where 'noise' not only means ambient and electronic noise, but also 'noise' from other acoustic paths. For example, the reflected field from neighboring layers without an angle of intromission, renders the angle of intromission 'invisible'. As a case in point, in this environment, reflection coefficient data from a time window that includes the mud-sand interface, e.g., Figure 8, cannot be used to determine if an intromission angle exists, since the high reflectivity of the mud-sand interface effectively 'fills in' the null in the reflected field. Thus, in order to observe the intromission angle, all other layers have to be windowed out. Therefore, the reflection time series are windowed around the water-sediment interface, see Figure 10, spanning here the upper $1.7 \mathrm{~m}$ of sediment. Even in these windowed data there are some low amplitude, but somewhat contaminating, contributions from the source coda which can be seen between the direct path, at $\sim 0.04 \mathrm{~s}$ and the water-sediment interface. In the first processing of the data (in 2017) the source coda was not removed. In this present work, the coda is removed by coherent subtraction (see Appendix B).

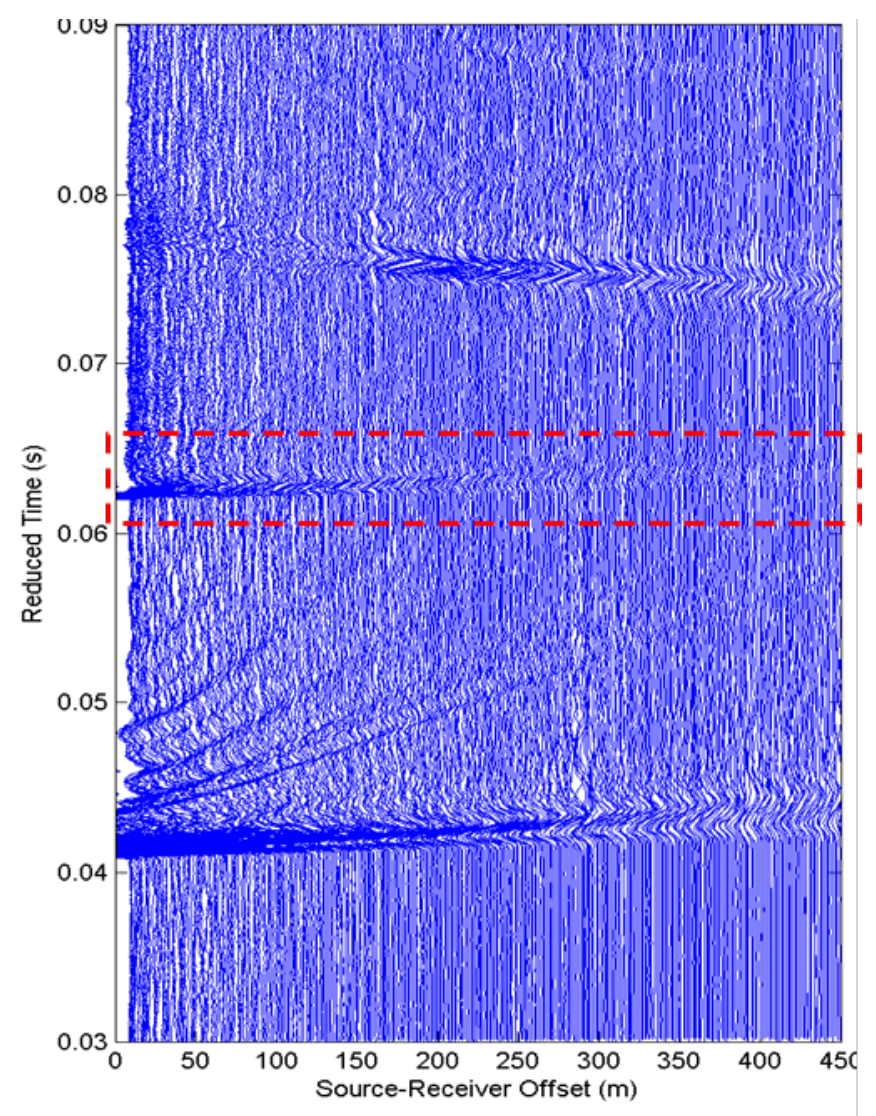


Figure 10. time series data from SWAMI site with window (red dashed lines) around the watermud interface in order to obtain the reflection coefficient averaged over the upper $1.7 \mathrm{~m}$.

The processed reflection coefficient data are presented in terms of bottom loss, $B L=-10$ $\log _{10}\left(\left|\hat{R}_{S}\right|^{2}\right)$ rather than $\left|\hat{R}_{S}\right|$ because it makes it easier to see the position of the angle of intromission, a peak in bottom loss, rather than a null in $|\mathrm{R}|$. If there is no peak in the bottom loss, then the sediment sound speed in the upper $1.7 \mathrm{~m}$ is the same or greater than that of the bottom water. If a peak is visible, then the sediment sound speed must be less than that of the bottom water. There is, in fact, a clear peak in the BL at $8^{\circ}$, see Figure 11a. This is the angle of intromission. The importance of this result is that this is a direct measurement - not an inversion, which proves beyond doubt the mud sound speed ratio must be less than unity. Thus, this is a valuable observation for the SBCEX17 community.
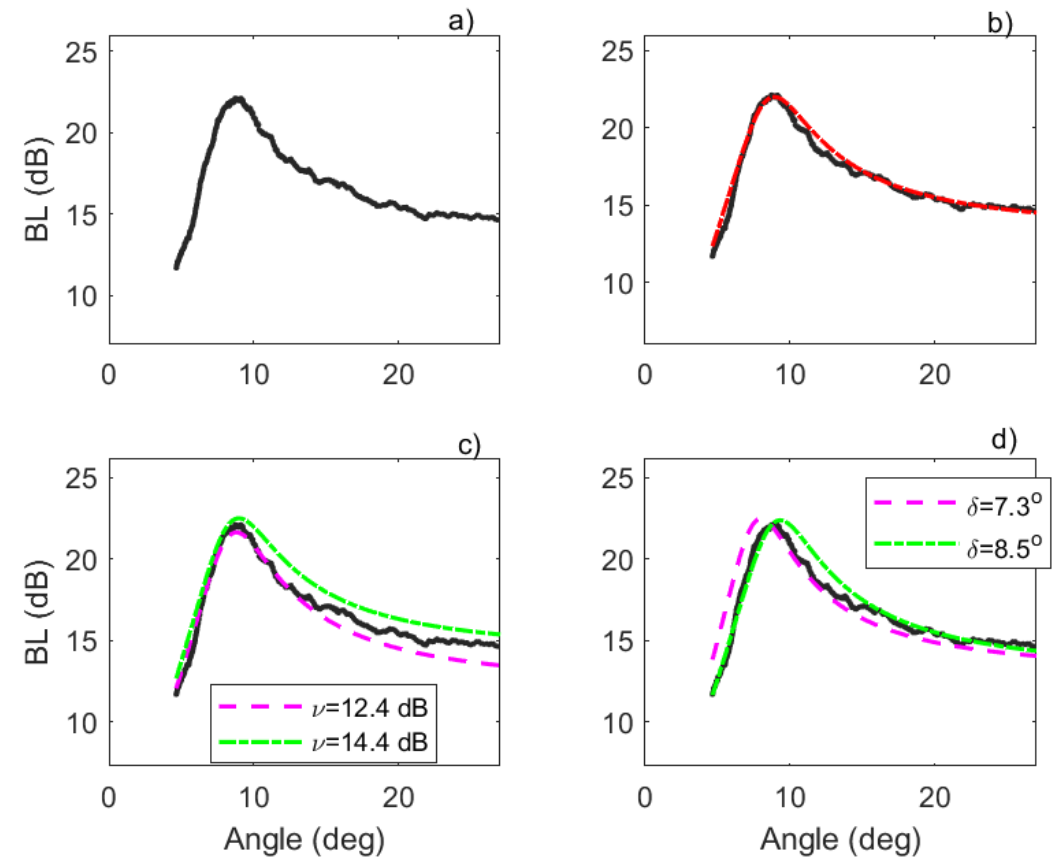

Figure 11. a) Measured reflection coefficient data at $1 \mathrm{kHz}$ at the SWAMI site, plotted as bottom loss, BL; b) measured data and model result (red) from the direct geoacoustic estimates; $c$ )-d) comparison of perturbed geoacoustic models provide an indication of geoacoustic uncertainty: $c)$ varying $v$ captures an extreme range of the measured reflection coefficient; d) varying

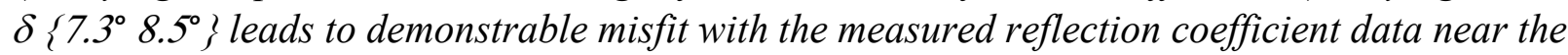
angle of intromission,

\section{i. Estimation of sound speed and density}

This result can be carried further. From the angle of intromission $\delta=8^{\circ}$ and $v=13.5 \mathrm{~dB}$ results in a density ratio of 1.557 and sound speed ratio of 0.9865 unambiguously obtained using Eq.(7)(8). Since the bottom water sound speed and density are known, this leads to a sediment density and sound speed of $1.598 \mathrm{~g} / \mathrm{cm}^{3}$ and $1454 \mathrm{~m} / \mathrm{s}$, averaged over the upper $1.7 \mathrm{~m}$ of sediment. As a sanity check, the directly obtained sound speed and density are used to predict bottom loss using Eq.(4), which, as expected, fits the observations quite well, see Figure $11 \mathrm{~b}$. 
It is of interest to determine the uncertainty of the sound speed and density estimates. The uncertainty depends upon both the measurement uncertainty and the estimation uncertainty. The measurement uncertainty of the angles was calculated via a Bayesian method from path travel time differences [16]. The measurement uncertainty at the angle of intromission, $8^{\circ}$, is $\pm 0.05^{\circ}$. Taking two standard deviations, 95\% probability, the sound speed and density ratio changes from the angle uncertainties $7.9^{\circ}$ to $8.1^{\circ}$ are [0.9875 0.9869] and [1.5323 1.5333], i.e., very small indeed. The measurement uncertainty of $v$ related to calibration is essentially zero since the reflection coefficient is self-calibrating, i.e., the measured reflected field is normalized by the measured direct path. There is also a measurement uncertainty of $v$ related to the source fluctuations which are a fraction of a dB.

A more significant contributor is the estimation uncertainty of $\delta$ and $v$. Somewhat crude uncertainty estimates can be made by varying these two parameters sufficiently to produce a clear mismatch between model and observations. For purposes here, this uncertainty metric will be loosely termed outer bounds. Variations in $\delta$ lead to significant changes in sound speed, but modest changes in density; variations in $v$ lead to significant changes in density and minor changes in sound speed. Perturbing the angle of intromission from $8^{\circ}$ to $\left\{7.3^{\circ} 8.5^{\circ}\right\}$ leads to concomitant changes in sound speed ratio of $\{0.9850 .989\}$. These can be seen in Figure $11 \mathrm{~d}$ to poorly match the measured angle of intromission compared to $\delta=8^{\circ}$ (Figure $11 \mathrm{~b}$ ). In other words, the sound speed ratio outer bounds $\{0.9850 .989\}$ indicate a rough outer limit on sound speed ratios that can explain the data. In Figure $11 \mathrm{c}$, a variation of about $\pm 1 \mathrm{~dB}$ in the reflection coefficient produces modeled BL at the extrema of the measured data with a density ratio outer bounds $\{1.491 .66\}$. These estimates provide a measure of uncertainty in sound speed and density from the observations. More rigorous uncertainty estimates will be made in follow-on work using statistical inference methods.

Nevertheless, these sound speed ratio and density ratio results are a significant contribution to the ongoing studies of mud properties at the NEMP. They provide a useful, direct observation to compare with other estimates which vary from $0.945-1.026$ over the band $25-1000 \mathrm{~Hz}$ (see (Ref [6], Fig. 7 which includes only sound speed ratio estimates at/near the water-mud interface). This large variation in sound speed, $120 \mathrm{~m} / \mathrm{s}$, cannot be explained by sound speed dispersion: a) since the frequency dependence in that figure is erratic, i.e., non-monotonic, and b) sound speed dispersion is modest in muds, e.g., [14]. The enormous variation in sound speed also cannot be ascribed to differences in location since: a) many disparate estimates (including the lowest estimate) were conducted in the central thick-sediment region and b) the geologic evidence indicates that "the very consistent lithology of Unit 1 [the upper few meters of mud] results in a very tight range of porosity and grain density values" across the NEMP experiment area [8].

\section{ii. comparison with a previous angle of intromission estimate}

Previous processing and analysis of the SWAMI angle of intromission data performed without coherent subtraction were first reported in 2017 [19] and shown here in Figure 16 (blue line). Note that the coda interference leads to bottom loss oscillations, rendering the angle of intromission position somewhat imprecise. For these bottom loss values (without coherent subtraction), the angle of intromission was estimated at $10^{\circ}$ in a band centered on $1 \mathrm{kHz}$; which 
yielded a sound speed ratio of $0.981 \pm 0.01$ as reported in the years 2017 [19] and 2020 ([6] Figure 7).

Coherent subtraction processing (see Appendix B) removes the coda interference, rendering the angle of intromission clear and unambiguous, see Figure 16 (red line). From the coherent subtraction result, the angle of intromission at $1 \mathrm{kHz}$ is $8^{\circ}$ with sound speed ratio of 0.9865 and narrower bounds of $\{0.9850 .989\}$.

\section{iii. can attenuation be inferred at the angle of intromission?}

The considerable value of the measuring the angle of intromission has been discussed above. It is of interest to further inquire if there is useful information about the compressional wave attenuation from the reflection coefficient at the angle of intromission, $\left|\hat{R}_{S}(\delta)\right|$. For a lossless fluid medium, $\left|R_{p}(\delta)\right|=0$ and increases monotonically with increasing attenuation. However, there are a number of reasons why the measured $\left|\hat{R}_{S}(\delta)\right| \neq 0$. First, the measurement is not a plane wave, but rather a curved wave front with a spectrum of angles, see Eq. (4). Thus, all plane-wave reflection coefficients within the Fresnel Zone contribute to the theoretical/measured $R_{s}$ at the specular angle $\delta$. In other words, even for a lossless fluid, all of the plane-wave reflection coefficients within the Fresnel Zone are non-zero, except at $\delta$, therefore $\left|R_{s}(\delta)\right| \neq 0$ except at infinite frequency or infinite source-receiver height. For example, for a lossless fluid mud the experiment geometry employed in these measurements and a frequency of $1000 \mathrm{~Hz}, B L(\delta) \sim 48 \mathrm{~dB}$, not infinity. Second, even though the shear wave velocity is small in muds, a few tens of $\mathrm{m} / \mathrm{s}$ [4], the conversion to shear waves adds a small loss and shifts the angle of intromission to slightly higher angles, of order $0.1^{\circ}$. Third, small-scale micro-layering, sediment heterogeneities, and interface roughness that normally are undetectable, may be detectable when the reflection coefficient is near zero. For example, in one study [20] it appeared that the effect dominating $\left|R_{S}(\delta)\right|$ was microlayering, but the effects could not be precisely modeled. Lastly, the fact that the attenuation is non-zero in the mud also leads to non-zero $\left|R_{s}(\delta)\right|$. Given the numerous factors affecting $\left|R_{s}(\delta)\right|$, can the compressional wave attenuation be extracted? To do so requires accurately accounting for each of the effects listed. The spherical wave effects can be modeled precisely and the losses due to the unknown shear velocity (profile) could at least be bracketed. However, properly accounting for the effects of (unknown) micro-layering, (unknown) sediment heterogeneities, and (unknown) interface roughness seems unlikely. Thus, estimation of attenuation from $\left|R_{S}(\delta)\right|($ or $B L(\delta))$ seems impractical, at least at present. The theoretical curve (red line) in Figure $11 \mathrm{~b}$ was computed with an attenuation of $0.32 \mathrm{~dB} / \mathrm{m} / \mathrm{kHz}$, which is an order of magnitude higher than that expected for a muddy sediment. Thus, this does not represent the effects of intrinsic attenuation but rather the small but perceptible effects of micro-layering and/or sediment heterogeneities on the reflection coefficient.

\section{Seabed reflection and scattering observations, 2-D}

As with the 1-D data, there is significant geoacoustic information content in the 2-D reflection data both in the time domain and in the frequency domain. An example of the match filtered time series data in Figure 12 shows this. The data are from the forward-most hydrophone at a pulse repetition rate of $1 \mathrm{~Hz}$ over a $100 \mathrm{~m}$ section of the March 30 upper East-West track. The pulse is a $250 \mathrm{~ms} 0.5-6 \mathrm{kHz}$ LFM, the Fresnel Zone diameter at $3 \mathrm{kHz}$ at the seabed is $\sim 6 \mathrm{~m}$ and the distance over ground between pings is $\sim 1.75 \mathrm{~m}$. Thus, two adjacent reflected arrivals share much 
of the same Fresnel Zone. Fast time in the plot (the y-axis) is arrival time and contains information about the vertical seabed characteristics. Slow time (x-axis) contains information about the lateral seabed characteristics. The first arrival shown in the plot at $\sim 0.72 \mathrm{~s}$ is the reflection from the water-sediment (mud) interface, the second arrival at $\sim 0.733 \mathrm{~s}$ is from the mud-sand interface. From the two-way travel time difference, $0.013 \mathrm{~s}$, the sediment thickness is $\sim 12 \mathrm{~m}$ (assuming a uniform $1500 \mathrm{~m} / \mathrm{s}$ and a seabed grazing angle of $45^{\circ}$ ).

There are three salient features in the data that are similar across the entire $70 \mathrm{~km}$ track at this source-receiver separation. First, the mud-sand interface has a high degree of lateral variability at short scales. For example between 20 and 35 s (UTC), the mud-sand interface is comprised of 2 or 3 closely spaced layers, but from 11-12 s (UTC) there is little if any evidence of an interface(s). Second, and by contrast, the water-mud interface at $0.72 \mathrm{~s}$ is very stable in amplitude and character from ping to ping. Third, above the mud-sand horizon, the mud is nearly devoid of 'coherent' layering, but has observable returned/scattered energy. In other words, adjacent signals between 0.72 and $0.733 \mathrm{~s}$ have little spatial correlation one with another indicating that the 'mud' is not homogeneous. The nature of the sediment heterogeneities that lead to this acoustic response are not known at present.

One question is why these data indicate stronger heterogeneities than in the chirp sonar data interpreted by Goff et al., [21], which show relatively clear horizons within the mud and relatively weak heterogeneities. The frequencies here are nearly the same as those in [21]. The most likely reason is that the source in [21] has a relatively narrow beampattern, which delimits the insonified volume, whereas the source is omnidirectional for these data.

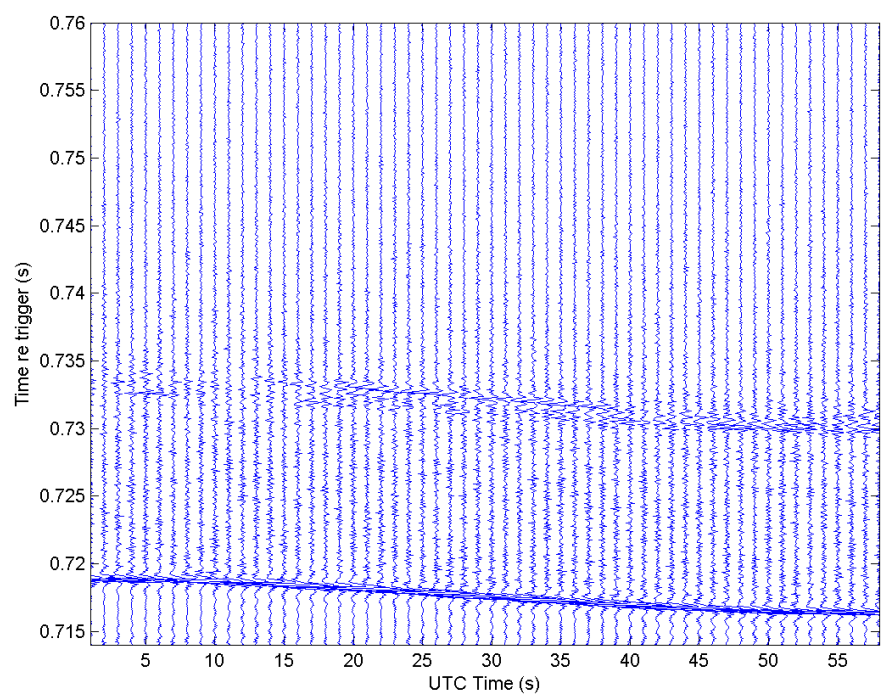

Figure 12. Match-filtered arrivals for a single channel on the cardioid array March 30, 2017 with a ping rate of $1 \mathrm{~Hz}$ and speed over ground $\sim 2 \mathrm{~m} / \mathrm{s}$. The earliest arrival, $\sim 0.72 \mathrm{~s}$, is from the watermud interface and the later arrival, $0.735 \mathrm{~s}$, is from the mud-sand boundary. The total distance covered on the seabed in this plot is about $100 \mathrm{~m}$. The location is about $70.58^{\circ} \mathrm{W} 40.47^{\circ} \mathrm{N}$ (on the upper leg of the racetrack, see Figure 3). The time re trigger is relative to an arbitrary time. The source-receiver offset is $\sim 30 \mathrm{~m}$ and grazing angle at the seabed is $\sim 45^{\circ}$. Note that the effects of towed array depth changes have not been removed. 

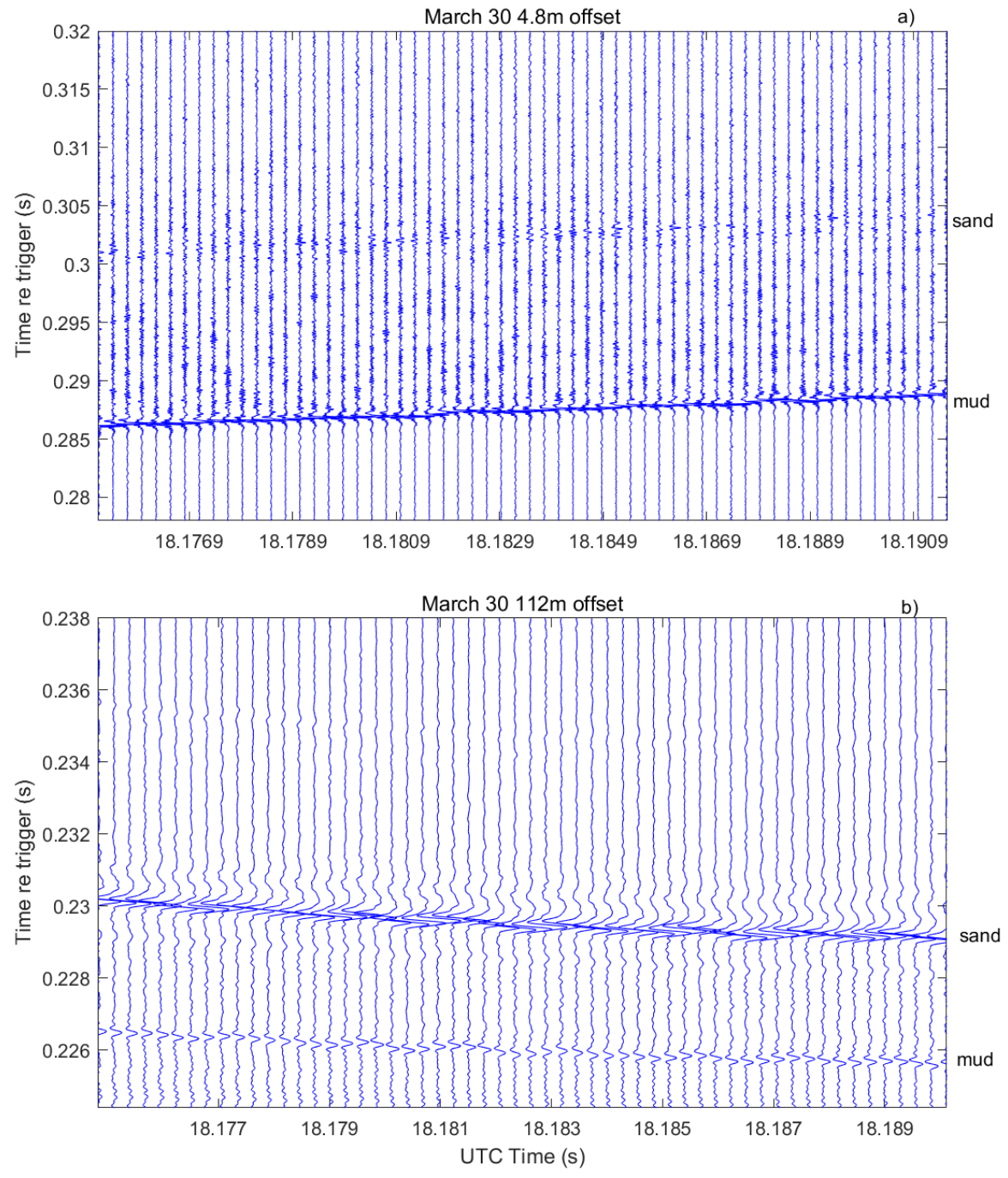

Figure 13. March 30, 2017 DSG seabed reflection data from source-receiver offset of a) $4.8 \mathrm{~m}$ where the water-mud reflection is at about $0.288 \mathrm{~s}$ and the mud-sand interface $\sim 0.3 \mathrm{~s}$ b) $112 \mathrm{~m}$ where the water-mud reflection is at about $0.226 \mathrm{~s}$ and the mud-sand interface $\sim 0.23 \mathrm{~s}$. Note that VIM and drogue depth changes have not been removed. The sensors in each plot have a slightly different clock and the time re trigger is arbitrary. The salient point is the difference in the character of the interface reflections and scattering from the sediment volume. The clock on the upper plot was adjusted (40 s) to roughly synchronize the two clocks, but at this stage of the analysis the inter-clock time error is still at least a few tenths of a second. The measurement location is $\sim 70.65^{\circ} \mathrm{W} 40.47^{\circ} \mathrm{N}$ (on the upper leg of the racetrack, see Figure 3).

The nature of the interface reflections and mud volume scattering is further explored in Figure 13 at two different angles on the seabed. The top plot is from a small source-receiver offset, $4.8 \mathrm{~m}$ (a grazing angle $\sim 81^{\circ}$ ) the bottom plot is from a source-receiver offset of $112 \mathrm{~m}$ or a grazing angle of $15^{\circ}$. One of the striking differences is that near normal incidence, Figure 13a, scattering from within muddy sediment volume is high, but the scattering is much smaller at $15^{\circ}$, Figure $13 \mathrm{~b}$. The higher scattering from the mud volume near normal incidence can also be seen clearly from the 
fixed-mooring data in Figure 5b, Note the 'noisy' time series between the mud and the sand boundaries between offsets of about $\pm 50 \mathrm{~m}$; this is clearly not noise from the passing ship, for example, since the 'noise' is not observed before the mud layer arrival. One candidate explanation is isotropic heterogeneities, however, these tend to be rare in nature. However, small heterogeneities where the effective radius is much, much smaller than the wavelength, e.g., some shell fragments, can be approximated as isotropic. Theory predicts, though that the scattered field from small heterogeneities decays more slowly with decreasing angle than the observations. For example, the ratio of the predicted scattered field at $45^{\circ}$ to that at normal incidence is only 0.8 ; the ratio of the scattered field at $15^{\circ}$ to that at normal incidence is only 0.6 . The scattering observed in the mud layer in Figure $5 \mathrm{~b}$ and Figure 13 decays with angle much faster than the decay from isotropic heterogeneities. Thus, heterogeneities much smaller than a wavelength do not seem to be a plausible explanation.

The scattering in the mud layer is however consistent with anisotropic heterogeneities, in which the lateral dimension is much larger than the vertical. This is a common characteristic of sediment volume homogeneities inasmuch as marine depositional processes tend to form sediments with larger lateral than vertical scales, sometimes orders of magnitude larger.

The presence of sediment volume heterogeneities within the mud unit is somewhat surprising given that time period in which the mud was deposited, the last $\sim 11,000$ years. During that time, this area has been in an unusually quiescent condition, i.e., a region in which oceanographic conditions permitted the deposition of very fine-grained sediments, essentially the only region along the entire mid-shelf of the US east coast. However, thin lenses of silt or sand could have been deposited from large storms. Though the volume heterogeneities are apparent acoustically in the mud unit, the extent of their impact on measuring dispersion is not known at this time. It should be noted that the mud-sand boundary was formed when the area was sub-aerially exposed; its roughness and complexity is less surprising.

Another aspect to note in Figure 13 is that the boundary reflection amplitudes are also quite different between near normal and grazing incidence. Near normal incidence, $81^{\circ}$, the mud-sand reflection is weak relative to the water-mud reflection. At low grazing angle, $15^{\circ}$, the situation is reversed. The primary explanation for this difference is that near normal incidence, the density ratio controls the reflection coefficient at both boundaries - the density ratio is $\sim 1.55$ at the watermud boundary and in the neighborhood of 1.2 at the mud-sand boundary. By contrast, at low angles, the reflection amplitude at the mud-sand boundary is controlled by the sound speed ratio, which leads to a critical angle. At the water-sediment interface, the reflection amplitude at low angles is controlled by both the density and sound speed ratio, and the proximity of the angle of intromission at $\sim 8^{\circ}$ (where $\left|\hat{R}_{S}\right|$ is very small) means that at $15^{\circ}$, the amplitude will be lower than at normal incidence (see Figure 11).

It is also clear from the data that the mud-sand reflection is much more stable from ping to ping at low angles than at high angles, see Figure 13. This is due to several factors, the scattering crosssection is smaller at low angles than at steep angles, the Fresnel zone size is larger at low angles and at low angles the critical angle limits the influence of complex sedimentary structure below it. 
The above plots provide examples from a few hydrophones. Geoacoustic estimation/inference methods in future research will exploit various observables from the data set. For example, the cardioid aperture and both DSGs will be used to obtain the spherical wave reflection coefficient $\left|R_{S}(\theta, f, \tau)\right|$ along the entire track. From these data, a sequential trans-dimensional Bayesian approach will infer 2-D (depth and range) geoacoustic properties and their uncertainties. The Image Source Method [22] is a computationally simpler approach to estimate sound speed depth and range dependence and will be applied to the same data. The data will also be used to measure the seabed scattering strength as a function of angle and frequency.

\section{Summary}

Measurements were conducted on the New England Mud Patch to provide information about the vertical and horizontal geoacoustic variability, in particular that of the fine-grained, or muddy layer. The main findings from the present study are:

1) that an angle of intromission exists. This is direct proof, without modeling or inversion, that the sound speed in the upper $1.7 \mathrm{~m}$ of sediment (mud) is less than that of the water column. To the authors' knowledge, these are the first angle of intromission measurements made anywhere on the continental shelf of North America. These data are useful, among other things, to inform reasonable values of sound speed ratio at the NEMP, which currently range $0.945-1.026$ from $40-1000 \mathrm{~Hz}$, (Ref [6], Fig. 7).

2) The angle of intromission permits a precise estimate of the sediment sound speed ratio 0.9865 with outer bounds $\{0.9850 .989\}$ in the upper $1.7 \mathrm{~m}$.

3) sediment heterogeneities exist within the mud layer which are likely anisotropic with a large horizontal to vertical scale.

4) there is clear evidence of a critical angle, both in the time and frequency domain. The time domain data unequivocally show that the critical angle is associated with the mud-sand boundary. The critical angle at the thin sediment site is $\sim 5^{\circ}$ larger than the thick sediment site.

\section{Acknowledgements}

The authors thank the Office of Naval Research Ocean Acoustics program for their support of this research. We also thank Captain Kent Sheasly and his outstanding officers and crew on the R/V Armstrong, the Seabed Characterization Experiment Chief Scientists David Knobles and Preston Wilson, and all of our colleagues in the SBCEX17 community.

\section{Appendix A. M18C-6 beampattern measurements}

M18C-6 source beam pattern measurements were conducted in the anechoic test facility at Penn State. All pulses were $2 \mathrm{~ms}$ in length, transmitted at 100 Vrms with a $10 \%$ Tukey window. The source was mounted mid-depth in the tank, $\sim 2.7 \mathrm{~m}$, separated from the receiving hydrophone by $3.16 \mathrm{~m}$, for a spreading loss of $10 \mathrm{~dB}$. Beam pattern measurements were performed using a single frequency tone and rotating the transducer $360^{\circ}$. The rotational rate $\sim 3^{\circ} /$ second with the transducer triggered at a rate of $10 \mathrm{~Hz}$ resulted in an angular resolution of $\sim 0.3^{\circ}$. Beam patterns were generated at frequencies of $2-15 \mathrm{kHz}$ in $1 \mathrm{kHz}$ steps in both the horizontal and vertical 
plane. These planes are referenced to the source orientation during the experiment, where the power connector was horizontal, see Figure $2 b$.

Measurements were performed on two M18C-6 sources with very similar results. The beampatterns were virtually omnidirectional in both planes at below a few kilohertz and had deviations up to a few $\mathrm{dB}$ at the highest frequency of the transmitted pulses during the field experiment, $6 \mathrm{kHz}$.

Only one source was used during the experiment and its calibration is shown here. The $6 \mathrm{kHz}$ beampattern in the horizontal plane is shown in Figure $14 \mathrm{a}$ where the beampattern is $0-2 \mathrm{~dB}$ lower in directions along the transducer axis, where the axis is defined by the connector. Figure $14 \mathrm{~b}$ shows that the transducer is virtually omnidirectional in the vertical plane. The lower frequencies all showed less deviation from omnidirectional in the horizontal and vertical than shown here.

a)

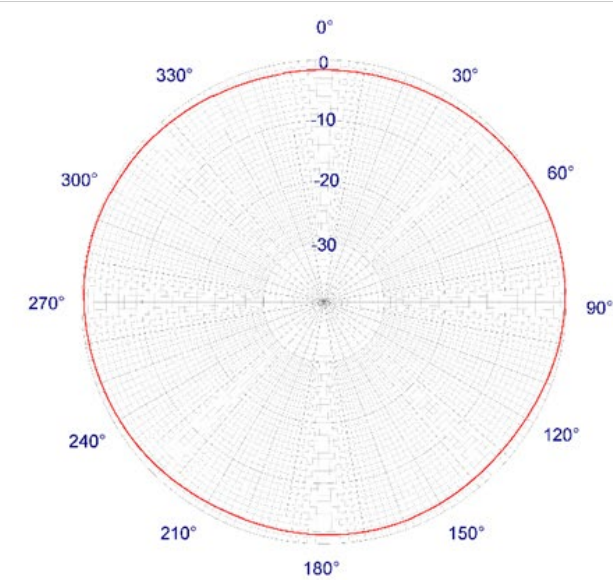

b)

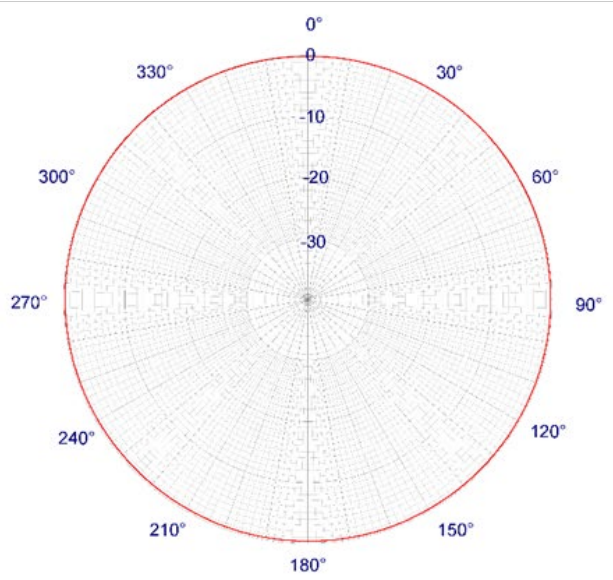

Figure 14. beampattern for the M18C source at $6 \mathrm{kHz}$ in the a) horizontal and b) vertical planes

\section{Appendix B. Coherent subtraction processing}

The uniboom source has a low frequency coda (see Figure 5a). The coda adds non-negligible noise within the time window for the angle of intromission measurement (where the reflection coefficient is near zero). In order to reduce the effects of the coda, coherent subtraction is performed using a reference (shallower) hydrophone.

Coherent subtraction for reducing a discrete scattered arrival from inside the towing platform (an Autonomous Undersea Vehicle) from seabed reflection data was discussed in [23]. This approach was broadly followed here with some differences. The pressure time series after coherent subtraction is defined as

$$
\hat{p}_{r}(t)=p(t)-a w(t) \mu\left(t-t^{\prime}\right)
$$

where $p(t)$ is the direct path and water-sediment interface reflected path from the deepest phone ( $\sim 11.5 \mathrm{~m}$ above the seabed), $a$ is the amplitude weighting, $w$ is the window function (7.5\% Tukey window $), \mu(t)$ is the reference time series, taken from the shallower phone $(\sim 15.5 \mathrm{~m}$ above the seabed), $t^{\prime}$ is the time shift to align the arrivals. 
Several options were considered for defining the amplitude, $a$, including using a) the direct path peak arrival and $b$ ) the root-mean-square amplitude of the direct path arrival including the coda. Since the amplitude of the coda (the removal of which is the objective of the processing) is not perfectly proportional to the direct path peak amplitude, the rms value of the direct path arrival including the coda was employed. The amplitude normalization on each ping used a time window delimited by the seabed arrival on the lowest phone.

In order to improve the accuracy of the time shift, $t^{\prime}$, the data were upsampled at 16 times the recorded sampling frequency, $32 \mathrm{kHz}$. Since the coda is slightly different for each ping, the reference time series $\mu_{i}(t)$ was taken for every ping $i$ and applied to $p_{i}(t)$. Results at several ranges are shown in Figure 15. In Figure 15a the source-receiver separation is $10 \mathrm{~m}$; note that the scaled and time-shifted hydrophones are nearly identical for the direct arrival $(3.7 \mathrm{~ms})$ and the coda $(5-$ $30 \mathrm{~ms}$ ) so that the coherent subtraction nearly completely removes the coda. In the early part of the direct arrival, the coherent subtraction is not zero, but this is of no consequence inasmuch as the purpose of the coherent subtraction is to reduce the coda near/at the bottom reflected arrival, $26 \mathrm{~ms}$. At this short offset, there is a $22 \mathrm{~ms}$ delay between direct and bottom reflected path and the coda has decayed to nearly zero at the seabed reflected arrival time. Thus, coherent subtraction yields only a minor correction. At a further source-receiver offset of $300 \mathrm{~m}$ (Figure $15 b)$ the time delay between the direct and bottom reflected is only $5 \mathrm{~ms}$ and the coda is not near zero (e.g., at $5.6 \mathrm{~ms}$ ) in the neighborhood of the bottom reflected path at $6 \mathrm{~ms}$. At these larger ranges (lower angles) coherent subtraction greatly reduces the coda, and hence improves the signal-to-noise ratio of the water-mud reflection coefficient.
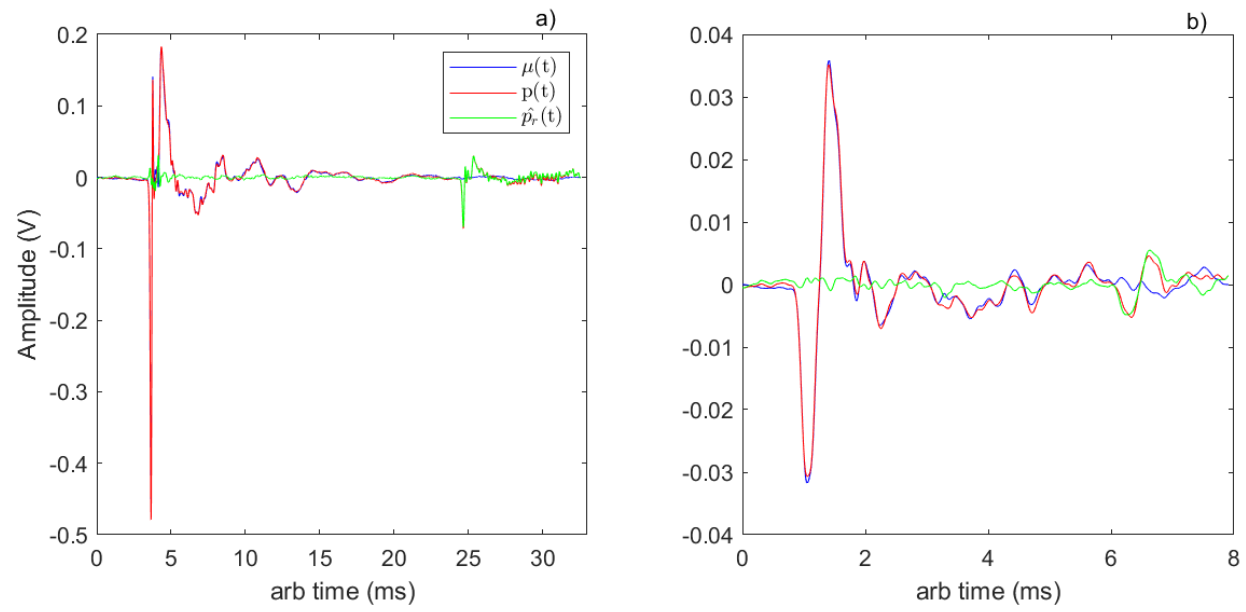

Figure 15. results of coherent subtraction, showing the reference (shallow) phone $\mu(t)$ (red); the deep phone $p(t)$ (blue), and the resulting coherent subtraction, $\hat{p}_{r}(t)$ (green), at source-receiver offsets of a) $10 \mathrm{~m}$ and b) $300 \mathrm{~m}$. The coda is seen in a) where the broadband direct path arrival $(3.7 \mathrm{~ms})$ is followed by a long low frequency coda, 5-30 ms. In b) the direct path arrival is $1 \mathrm{~ms}$.

Bottom loss without coherent subtraction is shown in Figure 16 (blue line). Note that since the coda has a dominant low frequency content, the coda interference increases with decreasing frequency. Also, since the coda amplitude is a decaying function, the coda interference increases 
with decreasing angle (where the time difference between direct and bottom reflected paths is shorter). Finally, the coda interference increases with increasing bottom loss, i.e., at very high bottom loss, the measurement will be dominated by the coda not the seabed reflection. It can be seen that coherent subtraction effectively removes the coda interference effects and thus reveals more accurately the position of the angle of intromission.
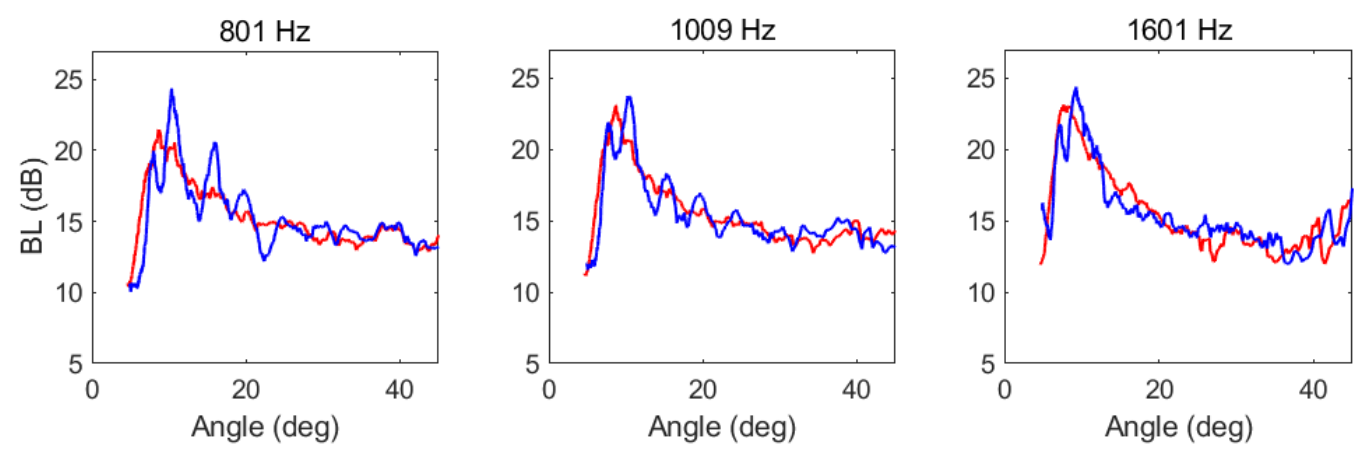

Figure 16. Bottom loss from the SWAMI site with (red) and without(blue) coherent subtraction. Data are averaged over a $1^{\circ}$ window.

\section{References}

[1] Pierce A. D., W. L. Siegmann, E. M. Brown, Characterization of mud sediments using the frequency dependence pf phase velocity and attenuation of compressional waves, Proc. Meetings Acoust., 31(1), 2017, [online] Available: https://asa.scitation.org/doi/abs/10.1121/2.0000891

[2] Biot M.A., Generalized Theory of Acoustic Propagation in Porous Dissipative Media, J. Acoust. Soc. Am., 34, 1254-1264, 1962.

[3] Hamilton E.L., Geoacoustic modeling of the seafloor, J. Acoust. Soc. Am. 68, 1313-1340, 1980 .

[4] G. R. Potty and J. H. Miller, Effect of shear on modal arrival times, IEEE J. Ocean. Eng., vol. 45, 103-115, 2020.

[5] Richardson, M.D., In-situ shallow water sediment geoacoustic properties, in Shallow water Acoustics, Ed. R. Zhang and J. Zhou, China Ocean Press, 1997.

[6] Wilson P., Knobles D., Nielsen T., Guest Editorial, An Overview of the Seabed Characterization Experiment, IEEE J. Oceanic Eng., 45,1-13, 2020.

[7] Bachman R.T., Acoustic and physical property relationships in marine sediment, J. Acoust. Soc. Am, 78, 616-621, 1985.

[8] Chaytor J.D., Ballard M.S., Buczkowski B.J., Goff J.Z., Lee K.M., Reed A.H., and A.A. Boggess, Measurements of geologic characteristics and geophysical properties of sediments from the New England Mud Patch, IEEE J. Ocean Eng., in review. 
[9] Lapinski A-L and D.M.F. Chapman, The effects of ignored seabed variability in geoacoustic inversion, J. Acoust. Soc. Am, 117, 3524-3538, 2005.

[10] Morley M.G., S.E. Dosso, and N.R. Chapman, Parameter Estimate biases in geoacoustic inversion from neglected range dependence, IEEE J Oceanic Eng., 33, 255-265, 2008.

[11] Seabed Characterization Experiment, Special Issue, IEEE J. Oceanic Eng., 45(1), 2020.

[12] Holland C.W., and J. Osler, High resolution geoacoustic inversion in shallow water: A joint time and frequency domain technique, J. Acoust. Soc. Am., 107, 1263-1279, 2000.

[13] Becker K.M. and J. Preston, The ONR Five Octave Research Array (FORA) at Penn State, in Proceedings of IEEE OCEANS'03, 2607-2610, 2003.

[14] Belcourt J., C.W. Holland, S.E. Dosso, and J. Dettmer, Depth-dependent geoacoustic estimates with dispersion from the New England Mud Patch via reflection coefficient inversion, IEEE J. Oceanic Eng.,45, 69-91, 2020, doi 10.1109/JOE.2019.2900115 (March 2019).

[15] Holland C.W., Seabed reflection measurement uncertainty, J. Acoust. Soc. Am., 114, 1861$1873,2003$.

[16] Belcourt J., S.E. Dosso, C.W. Holland, and J. Dettmer, Linearized Bayesian Inversion for Experiment Geometry at the New England Mud Patch, IEEE J. Oceanic Eng, doi 10.1109/JOE.2019.2900194

[17] Baron Rayleigh (John William Strutt), "The theory of sound", 2nd edition, 1896, as republished by Dover Publications, New York, Vol II, pg. 81, 1945.

[18] Holland C.W., Geoacoustic inversion for fine-grained sediments, J. Acoust. Soc. Am., 111, 1560-1564, 2002.

[19] Holland C.W., C.M. Smith, J. Belcourt, S.E. Dosso, Geoacoustic inferences from seabed reflection measurements on the New England Mud Patch (A), J. Acoust. Soc. Am., 2017.

[20] Holland C.W., Coupled scattering and reflection measurements in shallow water, IEEE J. of Ocean Eng., 27, 454-470, 2002.

[21] Goff J., J. D. Chaytor, A. H. Reed, G. Gawarkiewicz, P. S. Wilson, and D. P. Knobles, Stratigraphic analysis of a sediment pond within the New England mud patch: New constraints from high-resolution chirp acoustic reflection data, Mar. Geol., 412,81-94, 2018.

[22] Pinson S., L. Guillon, C. Holland, The Image-Source Method: a tool for geoacoustic inversion, Near Surface Geophysics, 18, 39-48, 2020. 
[23] Holland C.W., P.L. Nielsen, J. Dettmer, and S.E. Dosso, Resolving meso-scale seabed variability using reflection measurements from an autonomous underwater vehicle, J. Acoust. Soc. Am., 131, 1066-1078, 2012. 